\title{
Survey on graph embeddings and their applications to machine learning problems on graphs
}

\author{
Ilya Makarov ${ }^{\text {Corresp., 1, } 2 \text {, Dmitrii Kiselev }}{ }^{\text {Corresp., } 1}{ }^{,}$Nikita Nikitinsky ${ }^{3}$, Lovro Subelj $^{2}$ \\ 1 HSE University, Mocsow, Russia \\ 2 Faculty of Computer and Information Science, University of Ljubljana, Ljubljana, Slovenia \\ 3 Big Data Research Center, National University of Science and Technology MISIS, Moscow, Russia \\ Corresponding Authors: Ilya Makarov, Dmitrii Kiselev \\ Email address: iamakarov@hse.ru, dkiseljov@hse.ru
}

Dealing with relational data always required significant computational resources, domain expertise and task-dependent feature engineering to incorporate structural information into a predictive model.

Nowadays, a family of automated graph feature engineering techniques has been proposed in different streams of literature. So-called graph embeddings provide a powerful tool to construct vectorized feature spaces for graphs and their components, such as nodes, edges and subgraphs under preserving inner graph properties. Using the constructed feature spaces, many machine learning problems on graphs can be solved via standard frameworks suitable for vectorized feature representation.

Our survey aims to describe the core concepts of graph embeddings and provide several taxonomies for their description. First, we start with the methodological approach and extract three types of graph embedding models based on matrix factorization, random-walks and deep learning approaches. Next, we describe how different types of networks impact the ability of models to incorporate structural and attributed data into a unified embedding. Going further, we perform a thorough evaluation of graph embedding applications to machine learning problems on graphs, among which are node classification, link prediction, clustering, visualization, compression, and a family of the whole graph embedding algorithms suitable for graph classification, similarity and alignment problems. Finally, we overview the existing applications of graph embeddings to computer science domains, formulate open problems and provide experiment results, explaining how different networks properties result in graph embeddings quality in the four classic machine learning problems on graphs, such as node classification, link prediction, clustering and graph visualization.

As a result, our survey covers a new rapidly growing field of network feature engineering, presents an indepth analysis of models based on network types, and overviews a wide range of applications to machine learning problems on graphs. 


\title{
Survey on Graph Embeddings and its Applications to Machine Learning Problems
}

\author{
Ilya Makarov ${ }^{1,2}$, Dmitrii Kiselev $^{1}$, Nikita Nikitinsky ${ }^{3}$, and Lovro Šubelj ${ }^{2}$ \\ ${ }^{1}$ HSE University, Moscow, Russia \\ ${ }^{2}$ University of Ljubljana, Ljubljana, Slovenia \\ ${ }^{3}$ National University of Science and Technology MISIS, Moscow, Russia
}

Corresponding author:

First and second authors ${ }^{1}$

Email address: iamakarov@hse.ru,dkiseljov@hse.ru

\begin{abstract}
Dealing with relational data always required significant computational resources, domain expertise and task-dependent feature engineering to incorporate structural information into a predictive model. Nowadays, a family of automated graph feature engineering techniques has been proposed in different streams of literature. So-called graph embeddings provide a powerful tool to construct vectorized feature spaces for graphs and their components, such as nodes, edges and subgraphs under preserving inner graph properties. Using the constructed feature spaces, many machine learning problems on graphs can be solved via standard frameworks suitable for vectorized feature representation.

Our survey aims to describe the core concepts of graph embeddings and provide several taxonomies for their description. First, we start with the methodological approach and extract three types of graph embedding models based on matrix factorization, random-walks and deep learning approaches. Next, we describe how different types of networks impact the ability of models to incorporate structural and attributed data into a unified embedding. Going further, we perform a thorough evaluation of graph embedding applications to machine learning problems on graphs, among which are node classification, link prediction, clustering, visualization, compression, and a family of the whole graph embedding algorithms suitable for graph classification, similarity and alignment problems. Finally, we overview the existing applications of graph embeddings to computer science domains, formulate open problems and provide experiment results, explaining how different networks properties result in graph embeddings quality in the four classic machine learning problems on graphs, such as node classification, link prediction, clustering and graph visualization.

As a result, our survey covers a new rapidly growing field of network feature engineering, presents an in-depth analysis of models based on network types, and overviews a wide range of applications to machine learning problems on graphs.

Keywords: graph embeddings, network embedding, network representation, graph neural networks, graph convolutional networks, graph attention, node classification, link prediction, node clustering, community detection, graph classification, graph visualization, geometric deep learning, network science
\end{abstract}

\section{INTRODUCTION}

Many instances in the real world can be modelled as graphs or networks. Some of the typical examples include social interactions, biological data, such as protein interactions or neural connections, links between websites on the Internet, etc. One of the main goals of graph modelling is to formulate a general technique capable of processing structural data including relations between objects, which may also have some domain-specific information. For example, given a social network, we might be interested in predicting whether a pair of users are friends, or in identifying communities of interconnected users. The former leads to a link prediction problem on the graph, while the latter describes a node clustering problem.

We focus on graph representation theory, aiming to automatically learn low-dimensional vector features for the simplest graph motifs, such as nodes and edges, in a way that would enable efficiently solve machine learning problems on graphs including node classification, link prediction, node clustering,

PeerJ Comput. Sci. reviewing PDF | (CS-2019:04:37209:2:0:NEW 9 Dec 2020) 
while also tackling approaches for graph similarity and classification, and general aspects of graph visualization.

Before the emergence of the area, the extraction of important features for predictive tasks on graphs had to be manually engineered. It required a lot of efforts from the domain experts. For example, many approaches for graph representation rely on extracting summary statistics, such as vertex degrees or clustering coefficients (Bhagat et al. (2011)) popular in social sciences, graph kernels (Vishwanathan et al. (2010)) particularly used in computational biology to compute inner product similarities between graphs, or specifically designed features to measure neighbourhood similarity (Liben-Nowell and Kleinberg (2007)). In addition to the time-consuming feature engineering, such summaries were very inflexible, task/data-dependent, and did not generalize well across different prediction tasks on graphs. An alternative methodology is to learn feature representations automatically as an optimization problem. The goal is to design objective cost functions that capture dependencies and similarities in a graph while preserving high quality in relational machine learning tasks and constructing graph embeddings under efficiency constraints over time and memory.

Today, there exists a large variety of graph embeddings automatically extract vector representation for networks (Moyano (2017); Hamilton et al. (2017b); Cai et al. (2017); Cui et al. (2018); Goyal and Ferrara (2017); Chen et al. (2018a); Wu et al. (2019b)), knowledge graphs (Nickel et al. (2016)) and biological data (Su et al. (2020)). Some of these algorithms only work with structural information, such as popular Node2vec (Grover and Leskovec (2016)), LINE (Tang et al. (2015)), DeepWalk (Perozzi et al. (2014)), while others like GCN (Kipf and Welling (2016a)), GraphSAGE (Hamilton et al. (2017a)), VGAE (Kipf and Welling (2016b)) also use node attributes. The methods also differ based on whether a given graph is (un)directed, (un)weighted, (non-)attributed, (dis)assortative, if it changes over time in terms of adding/deleting nodes/edges, and whether they use a transductive or inductive approach for learning network dynamics inference. All of these models have their advantages and shortcomings, but what unifies them is the unique pipeline to verify the network embedding model in terms of the quality of machine learning tasks on benchmark datasets. In addition, authors measure construction and inference time efficiency, memory consumption, and a possibility to include graph dynamics in the model.

Most surveys on graph embeddings provide a simple taxonomy for graph models based on how the model is fitted and only show applications within the graph domain, e.g. node classification or link prediction (Moyano (2017); Hamilton et al. (2017b)). Goyal and Ferrara (2017) provide experiments and study the influence of hyperparameters on different tasks. Some works focus on a specific field such as attention models (Lee et al. (2019)) and graph neural networks (Wu et al. (2019b); Chen et al. (2018a); Zhang et al. (2018b)). Cui et al. (2018) compare models in terms of what information they preserve: structure and properties or side information. Neural network approaches are usually classified by the core architecture, e.g. recurrent neural networks (RNN) or convolutional neural networks (CNN), and losses for different tasks, such as cross-entropy for link prediction and node classification and reconstruction loss for unsupervised representation learning. Chen et al. (2018a) provides meta-strategies for choosing embedding models, but examine only deep learning based methods. Lee et al. (2019) follow the classification of Cai et al. (2017) and separate attention models by type of input and output, deriving recommendations for working with different graphs (heterogeneity, multi-view, directed acyclic graphs) and on different tasks (node classification, clustering, ranking, alignment, link prediction). Zhang et al. (2018b) is quite similar to other GNN surveys, but also provides an overview of modern models and tasks like reinforcement learning on graphs, analyses techniques for better representation learning like sampling strategies, skip connections, inductive learning and adversarial training.

In contrast, our work tries to generalize the advances of previous surveys. Our survey is not limited to specific model types and provides an overview from different angles: training process, input graph properties, specific tasks and applications in a non-graph domain, and open problems, etc.

The paper is structured as follows. We start with a brief explanation of general approaches to learn network embedding and introduce to a reader the core ideas of graph representation models. Next, we describe different models adapted to specific types of networks. Then, we state the most crucial machine learning problems on graphs and solutions to them based on network embeddings. To cover the use of overviewed models, we provide applications to other machine learning domains. We finalize review sections with the listing of open problems in the field of network representation learning.

Finally, we provide our experiments to understand in practice, how different graph embeddings perform on benchmark network datasets and interpret, why the chosen graph embedding model with a 
given training setting result in good or bad quality on a given benchmark dataset and how it is related to the method behind the model. Our experiment section aims to show how one can choose the best graph embedding by the nature of the model construction and network descriptive statistics, which is one the most interesting problems for practical applications of graph embeddings for machine learning frameworks.

\section{PRELIMINARIES}

Before describing any methods we need to introduce some definitions. We will use $V$ as a set of graph vertices, $E$ as a set of graph edges, $A$ as graph adjacency matrix and $G(V, E)$ as graph description. The procedure on constructing vector representation of a graph we are interested in is called graph embedding.

Definition 1 (Graph embedding) is a mapping from a collection of substructures (most commonly either all nodes, or all edges, or certain subgraphs) to $\mathbb{R}^{d}$. We will mostly consider node embeddings: $f: V \rightarrow \mathbb{R}^{d}, d \ll|V|$.

For many graph-based tasks, the most natural task formulation is unsupervised learning: this is the case when we need to learn embeddings using only the adjacency matrix $A$ containing information on structural similarity and possibly attributed features $X$, but without task-specific loss part. It is also possible that there are labels available for some substructures of the graph, and we wish to recover missing labels in a semi-supervised approach. One example of this is node classification, in which all nodes are available from the outset, but only a fraction is labelled.

Now let us clarify what is meant by a good embedding. By the embedding procedure, one should aim to compress the data, while retaining most of the essential information about similarities and simultaneously, extract important features from the structural information. What counts as essential may vary depending on an intended application; most common properties we want to capture in a graph are termed as node proximity and structural similarity (neighbourhood information and structural role, respectively).

Definition 2 (First and second order proximities) The first-order proximity describes the pairwise proximity between vertices. For any vertices, the weight $a_{i j}$ (possibly zero) of the edge between $v_{i}$ and $v_{j}$ characterizes the first-order proximity between these vertices, thus representing adjacency matrix $A=\left(a_{i j}\right)_{i, j=1}^{n}$. A neighborhood of vertex $v_{i}$ is defined as a set of adjacent vertices $N_{v_{i}}=\left\{v_{k} \mid a_{i k}>0, k \neq i\right\}$ thus meaning that vertex itself is not included in its neighborhood. The second-order proximity between a pair of vertices $v_{i}$ and $v_{j}$ describes the similarity measure between their neighborhood structures $N_{v_{i}}$ and $N_{v_{j}}$ with respect to a selected proximity measure.

\section{METHODS FOR CONSTRUCTING GRAPH EMBEDDING}

We briefly describe graph embedding methods of three general categories, corresponding to the perspective they take on embedding graphs: matrix factorizations, node sequence methods and deep learning based methods. These are, of course, not mutually exclusive, but it is more convenient to adhere to their primary features. We also cover a specific type of embeddings based on embedding space metric.

We select papers from several curated lists and major conferences on network science, artificial intelligence, machine learning and data mining, as well as core research publishers and indexing services. Paper sources are referred in Table 1. We used the following keywords: graph / network embeddings, graph / network representation, graph neural networks, graph convolutional networks, graph convolution, graph attention, graph / network classification / link prediction / clustering, deep learning for graphs, geometric deep learning, GCN, GNN, GAT.

Historically the first graph embedding methods were factorization based, which generally try to approximate a large matrix with a low-rank matrix factorized into a product of two matrices containing representations, thus modelling each entry of the original matrix with an inner product of representations. Sequence-based embeddings linearize the graph using random walks or diffusion and maximize the probability of observing the neighbourhood (context) of a node given its embedding. Deep learning-based models learn a function mapping a graph in the numeric form to a low-dimensional embedding by optimizing over a broad class of expressive neural network functions. 
Table 1. Paper sources

\begin{tabular}{|c|c|c|}
\hline Name & Link & Description \\
\hline \multicolumn{3}{|l|}{ Curated lists } \\
\hline by Rozemberczki & https://github.com/benedekrozemberczki/ & awesome-graph-classification \\
\hline by Soru & https://gist.github.com/mommi84/ & awesome-kge \\
\hline \multicolumn{3}{|l|}{ Conferences } \\
\hline Complex Networks & https://complexnetworks.org/ & International Conference on Complex Networks and their Applications \\
\hline IJCAI & https://www.ijcai.org/ & International Joint Conferences on Artificial Intelligence \\
\hline AAAI & https://www.aaai.org/ & Association for the Advancement of Artificial Intelligence \\
\hline ICML & https://icml.cc/ & International Conference on Machine Learning \\
\hline SIGKDD & https://www.kdd.org/ & Special Interest Group in Knowledge Discovery and Databases \\
\hline \multicolumn{3}{|l|}{ Domain conferences } \\
\hline ACL & http://www.acl2019.org/ & Association for Computational Linguistics \\
\hline Link Springer & https://link.springer.com/ & Online collection of scientific journals, books and reference works \\
\hline \multicolumn{3}{|l|}{ Indexing services } \\
\hline Scopus & https://www.scopus.com/ & Abstract and citation database \\
\hline Web of Science & https://www.webofknowledge.com/ & Citation Indexer \\
\hline Scholar Google & https://scholar.google.com/ & Web search engine for indexing full-text papers or its metadata \\
\hline
\end{tabular}

\subsection{Dimensionality reduction (matrix factorization) methods}

Definition 3 (Matrix factorization) is a decomposition of a matrix to the product of matrices. In this sense, the first matrix in series is named self node representation and the last matrix refers to node context.

Factorization models are common techniques in different machine learning domains to receive meaningful low-dimensional representation. Moreover, a lot of methods use similarity matrix between observations, which can also be reformulated as the graph similarity matrix.

Factorization techniques can be applied to a different graph representations and optimize different objectives. Some methods directly decompose the adjacency matrix A, e.g. MDS (Kruskal and Wish (1978)) reconstructs it by minimizing MSE between element $a_{i j}$ and euclidean distance between vectors $u_{i}$ and $u_{j}$ of manifold $U$. We can rewrite this with expression $\sum_{i=1}^{N} \sum_{j=1}^{N}\left(a_{i j}-\left\|u_{i}-u_{j}\right\|_{2}^{2}\right)^{2}$. LSI (Deerwester et al. (1990)) simply applies singular value decomposition to $A$ Golub and Reinsch (1971). In Wold et al. (1987) the manifolds are learned by maximizing variance for linear mixture. It is extended by LDA Martínez and Kak (2001).

Another way to use dimensionality reduction is to build proximity matrix of the graph. For example, IsoMap (Tenenbaum et al. (2000)) use shortest path matrix $D$ and apply MDS to learn embeddings. LLE (Roweis and Saul (2000)) learns node similarity by reconstructing weights matrix $W$ with which neighbouring nodes affect each other: $\left\|X-W^{T} U\right\|_{2}^{2}$ and repeats that procedure to learn manifold $U$ with achieved matrix $W$. LPP (He and Niyogi (2004)) estimates the weighted matrix $W$ as heat kernel and learn manifold $U$ by reduction of $W$ with Laplacian Eigenmaps technique. IsoMap and LLE were proposed to model global structure while preserving local distances or sampling from the local neighbourhood of nodes. The lower bound for methods complexity was quadratic in the number of vertices, still making them inappropriate for large networks.

Definition 4 (Graph Laplacian) If matrix $D$ is the diagonal degree matrix, i.e. $D=\operatorname{diag}\left(\sum_{j} A_{i j}\right)$, then Laplacian matrix can be defined as $L=D-A$.

Another approach for spectral graph clustering (Chung and Graham (1997)) was suggested in Belkin and Niyogi (2002) named Laplacian eigenmaps (LE), representing each node by graph Laplacian eigenvectors associated with its first $k$ nontrivial eigenvalues. The goal for Laplacian Eigenmaps class of models lies in preserving first-order similarities. Thus, a model gives a larger penalty using graph Laplacian if two nodes with larger similarity are embedded far apart in the embedding space. Laplacian objective function is symmetric in each pair $(i, j)$, and thus it cannot capture edge orientations. Kernel Eigenmaps (Brand (2003)) extends this approach to nonlinear cases. In contrast to LE, which preserved nodes dissimilarity, Cauchy embedding (Luo et al. (2011)) proposes optimization condition modification which preserves the 
similarity between vertices. Structure Preserving Embedding (SPE) (Shaw and Jebara (2009)) aims to use LE combined with preserving spectral decomposition representing the cluster structure of the graph. It introduces a new graph kernel and applies SVD to it.

Graph Factorization (GF) (Ahmed et al. (2013)) try to solve the scalability issue of factorization methods by decreasing node neighbourhood via graph partitioning and utilizing distributed computation.

The models in this class can be either symmetric and obtain final representations only from embedding matrix. GraRep (Cao et al. (2015)) consider $k$-hop neighborhood $\left(A^{k}\right)$ using SVD decomposition of $A^{k}$. HOPE (Ou et al. (2016)) is specific asymmetric transitivity preserving graph embedding. It is found that most asymmetric similarity measures can be formulated as $S=M_{g}^{-1} M_{l}$. Katz index refers to $M_{g}=I-\beta A, M_{l}=\beta A$. Rooted PageRank can be stated as $M_{g}=I-\alpha P, M_{l}=(1-\alpha) P$. Common neighbors is represented by $M_{g}=I, M_{l}=A^{2}$, and Adamic-Adar with $M_{g}=I, M_{l}=A \cdot D \cdot A$. To avoid calculation of similarity matrix authors propose to use generalized SVD and directly estimate matrices $M_{g}$ and $M_{l}$. Abu-El-Haija et al. (2017) proposed to use concatenation of two node representations capturing in- and out- connections. Authors of Wang et al. (2017d) proposed a Modularized Nonnegative Matrix Factorization (M-NMF) model to preserve the community structure in network representation. In ATP model (Sun et al. (2019b)) authors embed directed graph constructing two vectors for each node via factorization framework. Kefato et al. (2020) propose multi-objective framework for preserving directed nature of graph. SDNE (Wang et al. (2016)) uses autoencoders (as neural network based dimension reduction technique) to capture non-linear dependencies in local proximity.

Factorization based models are the best-studied theoretically and provide a well-known general framework for graph embedding optimization (Liu et al. (2019)), however, they suffer from high computational complexity for large graphs and often capture only a small-order proximity Perozzi et al. (2014).

\subsection{Sequence-based approaches}

Definition 5 (Random walk on graph) is a sequence of nodes obtained from the random process of node sampling. Usually, probability of choice of node $j$ after node $i$ is proportional to $A_{i, j}$.

Motivated by drawbacks of the matrix factorization approach, another approach emerged that attempts to preserve local neighbourhoods of nodes and their properties based on random walks (Newman (2005); Pirotte et al. (2007)). More specifically, the main idea is to maximize the probability of observing the neighbourhood of a node given its embedding, following the line of Skip-gram model initiated in NLP applications by Mikolov et al. (2013); Pennington et al. (2014). An objective of this type can be efficiently optimized with stochastic gradient descent on a single-layer neural network, and hence has lower computational complexity.

Definition 6 (Skip-gram) is method to learn sequence element $i$ representation via maximization of probability of elements in context of $i$ based on representation of $i$.

Two prominent examples of models in this class are node2vec (Grover and Leskovec (2016)) and DeepWalk (Perozzi et al. (2014)). DeepWalk performs a random walk over a graph and then uses sampled sequences to learn embeddings, using the Skip-gram objective (while having modifications for other NLP based sequence models, such as using Glove from Brochier et al. (2019)). Its predecessor LINE (Tang et al. (2015)) is equivalent to DeepWalk when the size of vertices' contexts is set to one. Node2vec extends the random walk with biasing parameters of BFS or DFS parameters. Another way of sampling based on diffusion was presented in diff2vec (Rozemberczki and Sarkar (2018)). By virtue of sampling being more centered around source nodes, it provides robust embeddings while being less flexible.

Walklets (Perozzi et al. (2016)) as a generalization of GraRep (Cao et al. (2015)) use weighted combination of embeddings of powers of adjacency matrix $A, A^{2}, \ldots, A^{k}$ to reduce the bias of Deepwalk for low-order proximities, and approximates computing $A^{i}$ by skipping nodes using short random walks (Perozzi et al. (2017)).

The focus on the local structure and non-convex optimization requiring the use of stochastic gradient descent and proper initialization limit random walk based methods in capturing the hierarchical structure of a graph. HARP (Chen et al. (2018b)) proposes a meta-strategy for graph embedding under recursive construction of nodes and edges into condensed graphs with similar global structure. These graphs are used as source initializations for embedding detailed graphs, resulting in the end in proper node and edge embeddings, which can be adopted for improving DeepWalk (Perozzi et al. (2014)), LINE 
(Tang et al. (2015)), and Node2vec (Grover and Leskovec (2016)) algorithms. It was further generalized for community preserving using Modularity Maximization (Tang and Liu (2009)) and supporting large free-scale networks (Feng et al. (2018)).

Alternatively, Struct2vec (Ribeiro et al. (2017)) uses structural similarity without using node or edge attributes but considering graph hierarchy to measure similarity at different scales. Liu et al. (2020c) uses rooted substructures of a graph to preserve structural similarity. Diffusion wavelet model to capture structural proximity was suggested in Donnat et al. (2018b). Another approach to control hyper-parameters in random-walk methods is Graph Attention (Abu-El-Haija et al. (2018)) learning multi-scale representation over adjacency matrix powers with the probabilistic approach for learning balancing weights for each power. It was further generalized to its deep learning analogue in Veličković et al. (2017) and Liu et al. (2018a), see also Lee et al. (2019) for details on attention models on graphs.

Extension of Deepwalk to heterogeneous networks was suggested in Metapath2vec (Dong et al. (2017)). Modifications of random-walk based methods using node attribute concepts and node proximities were suggested in GenVector (Yang et al. (2016b)). With GEMSEC (Rozemberczki et al. (2018)), the authors extend sequence-based methods with additional K-means objective encouraging clustering structure-preserving in the embedding space and improving overall performance. Discriminative Deep Random Walk (DDRW) (Li et al. (2016)) was suggested for the task of attributed network classification. Çelikkanat and Malliaros (2019) generalizes random walk based methods to the case of the exponential family of distributions for sampling strategies.

Sequence-based models, such as node2vec, can obtain high-quality embeddings of structural input graph by sampling node sequences and learning context-consistent embeddings but are not able to capture additional node/edge features while being transductive by their nature.

\subsection{Deep learning: graph convolutions}

Complex non-regular graphs structure makes graph filtering not as simply defined as on images. In the past decades, researchers have been working on the graph signal processing methods including filtering, wavelets, Fourier transformations using graph spectral domain. The studies on these methods can be found in Shuman et al. (2013); Ortega et al. (2018a).

Advances in deep learning have led to a new field of studies devoted to applying neural networks to graph data (Scarselli et al. (2009); Li et al. (2014a,b)). Recently, SDNE (Wang et al. (2016)) and DNGR (Cao et al. (2016)) use deep autoencoder to capture non-linearity in graphs and simultaneously apply dimension reduction for constructing graph embedding. SDNE use autoencoder preserving first order proximity and Laplacian Eigenmaps for penalizing long distances for embedding vectors of similar vertices. DGNR uses stacked denoising autoencoders over positive pointwise mutual information matrix obtained from similarity information based on random surfing. Both methods use global information and thus are not appropriate for large networks.

Kipf and Welling (2016a) propose Graph Convolutional Layer that offers a further simplified approximation to spectral convolution and achieves better computational efficiency for semi-supervised multi-class node classification is applicable for the other machine learning tasks. A model of several such convolutions is referred to as Graph Convolutional Network (GCN). Improvements over speed and optimization methods of training GCNs were suggested in Chen et al. (2017b, 2018c). Stochastic approaches for network embedding optimization were briefly over-viewed in Lei et al. (2018).

Assume the graph $G(V, E)$, adjacency matrix $A$ and feature matrix $X$ of size $\left(N_{\text {nodes }}, N_{\text {features }}\right)$, where $N_{\text {nodes }}$ refers to number of vertices and $N_{\text {features }}$ to number of node attributes. Then, GCN can be defined as set of hidden layers $H^{i}=\sigma\left(A H^{i-1} W^{i-1}\right)$ where $H^{0}$ is equal to matrix $X, W^{i}$ is learnable weight matrix. At the next hidden layer, these features are aggregated using the same propagation rule. It means that graph convolutions aggregate feature information of its neighbours based on the adjacency matrix. The idea of graph convolutions using spatial convolutions (operating with adjacency matrix) or spectral graph methods (operating with graph Laplacian) was proposed in Bruna et al. (2013); Duvenaud et al. (2015); Henaff et al. (2015); Niepert et al. (2016); Defferrard et al. (2016); Levie et al. (2017), while extending the GCN idea to recurrent models Li et al. (2015c); Monti et al. (2017b), mixture models of CNNs Monti et al. (2017a); Fey et al. (2018), diffusion convolutions Atwood and Towsley (2016); Li et al. (2017c), and models suitable for dynamic graphs under inductive learning paradigm Natarajan and Dhillon (2014); Hamilton et al. (2017a). All the methods suggest semi-supervised embedding, however, choosing unique labels for each vertex one may obtain an unsupervised version of network embedding. The GraphSAINT 
(Zeng et al. (2019)) provides a solution for scalability problem in training graph neural networks. It compares different topology-based sampling algorithms (node, edge and random walks) in terms of bias and variance of learned GCN model. It also introduces unbiased estimator for node aggregation.

Another idea is to use deep autoencoders to learn compressed representations that capture the essence of the graph structure. An autoencoder includes two nonlinear functions, an encoder and a decoder, and attempts to minimize reconstruction loss. One such model specifically designed for graphs is GAE, which consists of a GCN encoder (one or two stacked GCN layers in most use cases) that produces embeddings and an inner product decoder that reconstructs the adjacency matrix $\left(\hat{A}=\sigma\left(U U^{T}\right)\right.$, where $\sigma$ is non-linearity like sigmoid function and $U$ is embedding matrix of nodes). The weights of the model are trained by backpropagating the reconstruction loss, which is usually Mean Squared Error (MSE).

VGAE (Kipf and Welling (2016b)) is a probabilistic counterpart of GAE. It introduces a distribution over latent variables $Z$, with these variables being conditionally independent Gaussians given $A$ and $X$ with means $(\mu)$ and diagonal covariances $(\sigma)$ being parameterized by two GCN encoders (Kingma and Welling (2013)). As in the case of images, VGAE just adds KL-divergence term between conditional distribution $q(Z \mid X, A)$ and unconditional $p(Z) \sim N(0,1)$ to the loss. After node embeddings are reconstructed via random normal distribution sampling, i.e. $Z=\mu+\sigma \varepsilon$. Then adjacency matrix is decoded using inner product of achieved vector $Z$ as in simple GAE.

In very recent work, authors of GraphSAGE (Hamilton et al. (2017a)) offer an extension of GCN for inductive unsupervised representation learning and offer to use trainable aggregation functions instead of simple convolutions applied to neighbourhoods in GCN. GraphSAGE learns aggregation functions for a different number of hops that are applied to sampled neighbourhoods of different depths, which then are used for obtaining node representations from initial node features. PinSage (Ying et al. (2018a)) extends the previous algorithm with the importance sampling based on random walks. Importance score is calculated simply as visit counts. It provides better scalability and quality. GAT (Veličković et al. (2017)) use masked self-attention layers for learning weights balancing impact of neighbours on node embedding, and supporting both, inductive and transductive learning settings. In Liu et al. (2018a), authors suggested specific layers controlling the aggregation of the local neighbourhood over BFS and DFS sampling, thus generalizing Node2vec (Grover and Leskovec (2016)) model to graph neural networks. Similar to GCN, GAT contains several hidden layers $H^{i}=f\left(H^{i-1}, A\right)$, where $H_{0}$ is a graph node features. In each hidden layer linear transformation of input is firstly calculated with the learnable matrix $W$. The authors replace the adjacency matrix by learnable self-attention in form of a fully-connected layer with activation and further normalization with softmax. Generalization of gated recurrent graph neural networks ( $\mathrm{Li}$ et al. (2015c)) was suggested in Message Passing Neural Network (MPNN) (Gilmer et al. (2017)) providing a differentiable way to combine information from neighbours.

Nowadays, many advanced deep neural network models are adapted to graph data. Graph generative adversarial networks were suggested in Ding et al. (2018); Yu et al. (2018). In You et al. (2018), recurrent graph neural network was suggested for the task of graphs generation. Pooling operators for graphs were used in Defferrard et al. (2016); Ying et al. (2018b). Yuan and Ji (2020) modernize classic pooling to account graph structure using Conditional Random Fields. Adversarially regularized variational graph autoencoder (ARVGA) was suggested in Pan et al. (2019). Zhu et al. (2020a) develop the DGGAN model that jointly learns source and target vectors for the directed graphs employing adversarial techniques. Liu (2020) builds Anonymized GCN with adversarial training to be robust to the noise attacks. Hettige et al. (2020) propose the RASE model, that applies Gaussian denoising attribute autoencoder for achieving robustness of received embedding, while Laakom et al. (2020) catches the uncertainty by learning probability Gaussian distributions over embedding space. Weng et al. (2020) employs adversarial training for variational graph autoencoder. Zhu et al. (2020b) use node feature smoothing for learn better embeddings. Jing et al. (2020) designs variable heat kernel to learn robust representations.

Deep Learning models are now a study of vulnerability to adversarial attacks, in particular, it relates to structural data. The first approaches for detection of node/edge add/remove mechanisms were studied in Bojcheski and Günnemann (2018); Chen et al. (2018d), while other researchers focused on methods for unsupervised (Sun et al. (2018b)), semi-supervised (Chen et al. (2018e)) and supervised (Zügner et al. (2018)) scenarios of graph embedding construction, and application for ML problems. The black-box approach was formulated in Dai et al. (2018) and further covered in general overview for the problem of graph data poisoning (Chen et al. (2019b)) and its applications to social media data (Zhou et al. (2018)) and knowledge graphs (Zhang et al. (2019b)). A survey of methods for defence from adversarial attacks 
on graphs was suggested in Sun et al. (2018a).

The deep learning models propose a new way of approximation for classic graph convolutions and kernels, which allows extracting embeddings faster. A mixture of it with semi-supervised techniques gives the state-of-the-art results in terms of scalability, speed and quality on downstream tasks.

\subsection{Hyperbolic (non-Euclidean) embeddings}

The Euclidean space is not the best for structures like graphs, because has the low descriptive ability for hierarchical and scale-free structures. So, researchers have considered other space, that can successfully represent it in a comparatively low number of dimensions, saving the basic properties like angles. It allows using classical machine learning methods in down-streamed tasks.

In certain cases, embedding into non-Euclidean spaces may be beneficial for model performance (Kleinberg (2007); Shavitt and Tankel (2008); Krioukov et al. (2009)). LEs were also used for constructing embedding in hyperbolic space (Alanis-Lobato et al. (2016)). Deep learning approach was applied for hyperbolic embedding in Chamberlain et al. (2017).

There is no exact research on the properties of embedding spaces, but researchers mostly pay attention to preserving low dimensional space, catching graph properties and model quality trade-off.

\section{SPECIFIC EMBEDDINGS BASED ON NETWORK TYPES}

In this section, we show specific embedding models generalizing core methods of network representation to a certain domain of networks and applications based on the network type.

\subsection{Attributed networks}

Real-world networks are often accompanied with additional features for nodes and edges, such as labels, texts, images. These attributes tend to be correlated for close graph structures and could affect network embedding by adding additional information for the similarity of nodes. The attributes are usually represented by high-dimensional vectors of features (which are sparse for just label attributes). Once the attributes are represented by their embeddings, the task is to incorporate them in network embedding model (under unsupervised or semi-supervised framework).

The authors of TADW (Yang et al. (2015)) represent DeepWalk model as matrix factorization and incorporate text attributes into factorization framework. PLE (Ren et al. (2016)) jointly learns the representations of entity types and links together with text features. In Le and Lauw (2014), a generative model for document network embedding was suggested based on topic modelling of documents using Relational Topic Model (RTM) (Chang and Blei (2009)) and the relationships between the documents. In Ganguly et al. (2016), authors combine text and network features for co-authorship recommendations.

Augmented Relation Embedding (ARE) (Lin et al. (2005)) adds content-based features for images using graph-Laplacian spectral embedding modification. In Geng et al. (2015); Zhang et al. (2015, 2017), authors suggested to embed images, textual and network information for modelling user-image interaction.

In addition to structural similarity, in certain cases feature similarity may be also important. Twolayered network embedding for node-to-node and text-to-text similarities was suggested in Sun et al. (2016). In Zhang et al. (2016b), the authors proposed the HSCA model, embedding homophily, network topological structure and node features simultaneously. In DeepBrowse (Chen et al. (2017a)), the authors suggested using DeepWalk-based node similarity together with priority ranking for recommender system based on an interaction graph. Label preserving attribute node embedding was suggested in Tri-party Deep Network Representation (Pan et al. (2016)). Modifications of random-walk based methods using node attribute concepts and node proximities were suggested in GenVector (Yang et al. (2016b)).

Label attributes are also an important part for such problems as classification of nodes and edges, or community information (assigning each node a community label). Community preserving network embeddings were suggested in Shaw and Jebara (2009); Wang et al. (2017d); Rozemberczki et al. (2018). Incorporating group information was presented in GENE model (Chen et al. (2016b)) under a supervised framework.

Semi-supervised frameworks for learning network embedding under loss constraints for labeled data were suggested in Planetoid Yang et al. (2016a), Max-margin Deep Walk (Tu et al. (2016)) and LANE (Huang et al. (2017)). 


\subsection{Heterogeneous networks}

A heterogeneous network presents a different concept of graph representation, in which nodes and edges may have different types (or even multiple edges).

The heterogeneous network embeddings either learn embeddings in the same vector space (Li et al. (2015a); Zhao et al. (2015)), or construct the embeddings separately for each modality and then aggregate them into one space, such as HNE model (Chang et al. (2015)) and Tang and Liu (2011), or even aggregate over multiple network layers (Xu et al. (2017)) or different relation features (Huang et al. (2017)).

Random-walk based approach for different node types based on DeepWalk was presented in Metapath2vec (Dong et al. (2017)). Similar approaches based on meta-path random walks for graph embedding were suggested in Huang and Mamoulis (2017); Chen and Sun (2017). Jacob et al. (2014) use heterogeneous network embedding for node classification across different node types. A similar problem was posed for author identification on double-blind review scenario (Chen and Sun (2017)). Study by Jiang et al. (2020) provides a framework for efficient task-oriented skip-gram based embeddings. Hu et al. (2019) utilizes the generative adversarial networks, which learn node distributions for efficient negative sampling. Shi et al. (2020b) proposes a method for automatic meta-path construction.

Cao et al. (2020) use the graph attention mechanism for heterogeneous graph embedding task. MAGNN architecture (Fu et al. (2020)) extends simple attention mechanism with several levels: node attributes, inter meta-path information and intra meta-path semantic information. DyHAN (Yang et al. (2020)) presents the model for dynamic heterogeneous graphs with hierarchical attention. Another way to use the attention mechanism in dynamic heterogeneous networks is the Li et al. (2020). It employs three types of attention: structural, semantic and temporal.

Heterogeneous graph embeddings are widely used in real-world applications. Hong et al. (2020) estimates the arrival time for transportation networks, Ragesh et al. (2020) use it in text classification. Chen and Zhang (2020); Li et al. (2020) utilizes HIN embedding for multi-modal data fusion task. Zhang et al. (2020) preserves the relationships in HIN. A survey on heterogeneous networks can be found in Wang et al. (2020).

\subsection{Signed networks}

In a signed network, each edge is associated with its weight, taking values from the set $\{1,-1\}$, which usually represents belief or opinion sentiment for different relations types. These networks are specifically considered apart from Heterogeneous networks as important objects for social network analysis, although they are still just a specific type of such networks. One of the tasks on such networks is predicting links and their signs (Liu et al. (2015a)).

SiNE (Wang et al. (2017c)) is a DNN model aiming at close relationships with friends (positive weight) rather than with foes (negative weight). For highly positive social networks a virtual node with negative relation is proposed to use in the model, which uses pairwise similarities optimization under constraint mentioned above. In Yuan et al. (2017), the authors propose a local neighbourhood aggregation model SNE for each type of positive and negative relations. Kim et al. (2018b) propose random-walks based model SIDE for signed directed networks. Also, they provide socio-psychological interpretation for each term in the loss function. SIGnet (Islam et al. (2018)) develops new target node sampling for more efficient learning. In oppose to previous works, Lu et al. (2019) provides signed network embedding powered by Status Theory Leskovec et al. (2010). It natively works with directed networks by preserving node ranking except direct node similarity.

\subsection{Multi-layer networks}

Multi-layer networks are used to model complex systems with different levels of interaction between nodes, e.g. whole Airline network with different carriers. Each layer in such networks corresponds to different types of relationships.

Liu et al. (2017a) compare three aggregation methods for single-layer network embedding models: merging of different layers in one network, single-layer vectors concatenation and between-layer random walks. The best results show the last method named layer co-analysis because it allows learning betweenlayer interactions. In Xu et al. (2017) authors provide an example of coupling into joint space two separately learned heterogeneous networks embeddings. IONE Liu et al. (2016) preserves users similarity based on their followers and followees for several social networks. A hierarchy-aware unsupervised node feature learning approach for multi-layer networks was proposed in Zitnik and Leskovec (2017).In Li 
et al. (2018) authors develop the single optimization framework for both within-layer and between-layer communication. It exploits spectral embedding and the block model.

\subsection{Temporal networks}

A lot of real-world networks are evolving over-time. Most of the described above methods concentrate on the static embeddings, so it works poorly in the temporal scenario.

Haddad et al. (2019) propose the adaptation of Node2vec model to the dynamic case. Authors also introduce the task-specific temporal embeddings. Rossi et al. (2020a) provide the generic framework named Temporal Graph networks for deep learning on dynamic graphs. Fathy and Li (2020) apply the graph attention to the temporal networks. Zhong et al. (2020) develop the model for efficient community mining. Rokka Chhetri and Al Faruque (2020) present the model for dynamic physics graphs. CTGCN model (Liu et al. (2020a)) generalizes graph convolution networks with feature transformation and aggregation. It builds the hierarchical representation of the graph with K-cores and applies GCN to it. Goyal et al. (2020) use the recurrent neural networks to catch the dynamics. There is one more specific graph type: temporal interaction networks, such as user-item interactions in the recommender systems. Zhang et al. (2020b) creates the embedding approach for such graph utilizing coupled memory networks.

Nowadays, methods based on smart neighborhood aggregation, such as limiting random walks over clusters Chiang et al. (2019) and precomputing diffusion-based neighborhoods for one-layer GCN Rossi et al. (2020b) show great performance over existing approaches, thus combining advances in deep learning and neighborhood sampling methodology.

\subsection{Large graphs}

We have already mentioned that random walks and graph neural networks were proposed as the approximations for the different classic matrix factorization techniques. So in this section, we will discuss approaches to scale up GNN training.

The basic idea implemented in different papers is a sampling. GraphSAGE (Hamilton et al. (2017a)) learns trainable aggregations for sampled node neighbourhood. This approach was further improved with fixed-length random walk based importance sampling of the neighbourhood in Ying et al. (2018a). GraphSAGE also provides the idea of minibatch training for GNNs. A similar idea was proposed in the Chen et al. (2018c). Salha et al. (2020) propose to use linear aggregation over direct neighbours to simplify computations. The GraphSAINT (Zeng et al. (2019)) compares different topology-based sampling algorithms (node, edge and random walks) in terms of bias and variance of learned GCN model. It also introduces unbiased estimator for aggregation of node and normalizes propagation by this value, that solves the scalability problem.

Nie et al. (2020) is based on the idea of Locality Preserving Projection. It works with anchor-based proximity matrices and calculates these anchors via Balanced and Hierarchical K-means. Such an approach allow to reduce complexity from $n^{2} d$ to $n d m$ where $n$ is a number of samples, $d$ is embedding dimension and $m$ is a number of anchors. Akyildiz et al. (2020) extends the VERSE (Tsitsulin et al. (2018)) with graph partitioning and coarsening to provide fast embedding computation on the GPU. Atahan Akyildiz et al. (2020) analyzes effects of graph coarsening on different embeddings in comparison to GOSH. Another distributed training framework was presented in Zheng et al. (2020a). It also provides efficient graph partitioning schemes for reducing between-machine communication. Gallicchio and Micheli (2020) keeps the graph embedding as the dynamical systems and study the embedding stability issue. Authors found that stable initialization allows to left weights untrained in deep sparse networks. Lu and Chang (2020) use softmax clustering for modularity maximization. They show that such a method is a linear approximation for main eigenvectors.

\section{APPLICATION OF GRAPH EMBEDDINGS TO MACHINE LEARNING PROB- LEMS}

Here, we aim to overview core machine learning problems involving structural data. We start with problems related to small graph motifs such as nodes and edges, while further going to the problems connected to subgraphs and graphs as a whole. 


\subsection{Node classification Definition 7 (Node classification) For a given graph $G(V, E)$ with known labels for some of nodes from $V$, node classification is the task of predicting missing labels for existing or newly added nodes.}

Node classification deals with assigning class labels to nodes based on labelled nodes data (Zhu et al. (2007); Bhagat et al. (2011)). The structural information is used in a context that "similar" nodes should have the same/similar labels. The original framework uses label propagation based on random walks statistics (Xiaojin and Zoubin (2002); Azran (2007); Baluja et al. (2008)). In an unsupervised framework, each node is embedded in a low-dimensional space following by training a classifier on the set of labelled node embedding vectors (Lu and Getoor (2003); Bhagat et al. (2009)). Authors use such machine learning models as logistic regression (Perozzi et al. (2014); Pimentel et al. (2017)), SVM (Wang et al. (2016, 2017d)), kNN (Le and Lauw (2014); Wilson et al. (2014)), random forest and xgboost (Makarov et al. $(2018,2019 \mathrm{c})$ ); the choice is usually made based on the size of training data, interpretability of features and embedding dimension.

In semi-supervised framework, node embeddings are learned via loss function containing regularization for labelled data predictions, penalizing "similar" nodes to have different labels (Li et al. (2016); Yang et al. (2016a); Tu et al. (2016); Kipf and Welling (2016a); Monti et al. (2017a)). Zhang et al. (2020) proposes hierarchical GCN and pseudo-labeling technique for learning in scarce of annotated data. Liu et al. (2020b) proposes a sampling strategy and model compression for handling sparsity of labels. Chen et al. (2020) employs contrastive learning techniques to achieve semi-supervised parametrized fusion of graph topology and content information. Zhu et al. (2020c) also use metric learning approach but applies it to corrupted graph substructures. Nozza et al. (2020) use two-phase optimization for attributed graph embedding. Shi et al. (2020a) aligns topology of attribute content network to the corresponding graph to simultaneously learn good embeddings. Wang et al. (2020) propose two models for the imbalanced scenarios. A survey on classic techniques for node classification can be found in Bhagat et al. (2011).

\subsection{Link prediction}

Definition 8 (Link prediction problem (LPP)) is a task of completing missing edges in noisy graphs or predicting new edges in temporal network structures. Formally, LPP for given graph $G(V, E)$ with adjacency matrix $A$ is a task of learning such function $f$ that reconstruct or predict next adjacency matrix $\tilde{A}$ based on different graph features such as metrics (e.g. Jaccard, Adamic-Adar), graph embeddings.

Network science approach to the problem of predicting collaborations results in the link prediction (LP) problem (Liben-Nowell and Kleinberg (2007)) for temporal networks and missing edges reconstruction in noisy network data. Basically, it is a method to apply standard machine learning framework for graph data considering feature space consisting of pairs of nodes and their features.

One of the interesting research questions is in the way of constructing edge embedding in a non-direct combination of node embeddings, as it was suggested in component-wise embeddings (Grover and Leskovec (2016)) or bi-linear combination of compressed node embeddings suggested in Abu-El-Haija et al. (2017). Certain practical applications for drug combinations was suggested in Zitnik et al. (2018).

HARP Chen et al. (2018b) incorporates several hierarchical layers while transmitting information from edge embedding to node embedding. Other systems of directly incorporating edge features and labels were suggested in CANE (Tu et al. (2017)) and LANE (Huang et al. (2017)). Models of joint node and edge structure learning were proposed in Dual-Primal GCN (Monti et al. (2018)) and ELAINE (Goyal et al. (2018)). A model for embedding event graphs in which event is described by several edges was presented in HEBE (Gui et al. (2016)). Wu et al. (2020) presents random walk with restart index. Phuc et al. (2020) embeds several graphs with similar structural properties to boost link prediction accuracy. Keser et al. (2020) employs skip-connections in VGAE.

Link prediction models are applied in web linking (Adafre and de Rijke (2005)), social dating services (Backstrom and Leskovec (2011)) and paper recommender system for digital libraries (He et al. (2010)). The reader can found an up-to-date survey in Srinivas and Mitra (2016).

LPP was specifically formulated in Liben-Nowell and Kleinberg (2007) based on nodes pairwise similarity measures. Approaches for link prediction include similarity based methods (Adamic and Adar (2003)), maximum likelihood models (Clauset et al. (2008)), and probabilistic models (Getoor and Taskar (2007); Heckerman et al. (2007)). In Tang and Liu (2012), authors are suggesting unsupervised approach for LP problem. Gao et al. $(2011,2015)$ suggested temporal link prediction based on matrix factorization 
technique and noise reduction in large networks. Attribute-based link formation in social networks was studied in McPherson et al. (2001); Robins et al. (2007), while deep learning approaches were presented in Liu et al. (2013); Zhai and Zhang (2015); Berg et al. (2017). Heterogeneous graph link prediction for predicting links of certain semantic type was suggested in Liu et al. (2017b, 2018b). An evaluation of link prediction models based on graph embeddings for biological data was presented in Crichton et al. (2018).

Two surveys on link prediction methods describing core approaches for feature engineering, i.e. Bayesian approach and dimensionality reduction were presented in Hasan and Zaki (2011); Lü and Zhou (2011). Survey on link prediction was published in Wang et al. (2015).

\subsection{Node clustering}

Definition 9 (Node clustering or community detection or graph partitioning) is the task of the partitioning of a graph $G(V, E)$ into several subgraphs $G_{i}\left(V_{i}, E_{i}\right)$ with a dense connection within groups and sparse connection between clusters.

Node clustering (also known as community detection in social network analysis) aims to find such a grouping (labelling) of nodes so that nodes in the same group are closer to each other rather than to the nodes from outside of the group (Malliaros and Vazirgiannis (2013)). No labels are provided on initial step due to unsupervised type of the problem. Methods use attribute (Zhou et al. (2009)) or structural information. The latter methods of graph clustering are usually based on either community detection (Newman and Girvan (2004); Fortunato (2010)) or structural equivalence (Xu et al. (2007)). In community detection (Shi and Malik (2000); Ding et al. (2001)), the cluster is defined as dense subgraph with a high number of edges inside subgraph, and a low number of edges between subgraph and the rest of a graph.

The general idea is to use node embeddings as a compressed representation of sparse graph adjacency matrix and then apply standard clustering algorithms, such as K-means or DBScan, for vectorized data (White and Smyth (2005); Tian et al. (2014); Cao et al. (2015); Chen et al. (2015b); Cao et al. (2016); Nie et al. (2017b)). Going further, joint optimization of clustering and node embedding was suggested in Tang et al. (2016b); Wei et al. (2017). Efficient iterative community aware network embedding was proposed in Wang et al. (2017d) and several others (Zheng et al. (2016); Cavallari et al. (2017)).

Teng and Liu (2020) propose multi-objective evolutionary algorithm for community detection. Zhang et al. (2020a) use multi-objective matrix factorization over several shortest path graphs and utilizes (MOEA) to find community structure. Salim et al. (2020) train the embeddings on different views for preserving many properties of a given network. Quiring and Vassilevski (2020) employs hierarchical coarsening of the graph to better extract clusters.

\subsection{Subgraph (and graph) embedding}

While studying network embedding, one may think of a way to aggregate or generalize low-level node feature representation to the whole network representation, thus stating the problem of embedding the whole graph (Song (2018)). Such vector is required for the graph-level tasks like graph classification, similarity and clustering. It considers the whole network as one structural unit in the training dataset.

The task is relevant to chemistry or biology domains (Nikolentzos et al. (2017); Zhang et al. (2016a); Duvenaud et al. (2015); Dai et al. (2016); Niepert et al. (2016); Kearnes et al. (2016)). They can also be applied for graph reasoning (Li et al. (2015c)) or computer vision tasks (Bruna et al. (2013)).

In Duvenaud et al. (2015), the sum based approach over network embedding was suggested. Following by it, in Dai et al. (2016), authors proposed neural network aggregation for constructing network embedding which is an argument for summing over subgraph nodes. Improvement of these methods was later suggested in Bronstein et al. (2017) based on approximations of spectral graph decompositions. Ordered-based (Niepert et al. (2016)) and fuzzy-based (Kearnes et al. (2016)) approaches based on aggregating features from convolutional approaches further improved subgraph embedding models. Sun et al. (2019a) maximize the mutual information between embedding and different graph substructures.

The general approach of Gilmer et al. (2017) as well as other convolutional approaches can be generalized by pooling-aggregation models or, as was suggested in Scarselli et al. (2009), by adding super-node for whole graph embedding. The attention mechanism was applied to the graph classification task (Lee et al. (2018)).

Definition 10 (Line (dual) graph) For a graph $G=(V, E)$ defined as a set of vertices $V$ and a set of edges $E \subseteq V \times V$ without loops and multi-edges we denote by $G^{*}=\left(V^{*}, E^{*}\right)$ a dual (Line) graph the 
nodes of which are the edges of $G$ and edges are nodes, in the sense that two adjacent nodes are connected by an edge if corresponding edges have a common node incident to them.

In graph-level tasks, specific network properties play a major role. So vectors reconstructing sophisticated similarity metrics closely related to the problem of graph isomorphism was studied in several works(Shervashidze et al. (2011); Niepert et al. (2016); Mousavi et al. (2017); Yanardag and Vishwanathan (2015); Narayanan et al. (2016)). GL2VEC (Chen and Koga (2019)) extends Narayanan et al. (2016) model with edge features by utilizing the line graph. The works on matching node embedding and graph kernels were suggested in Johansson and Dubhashi (2015); Nikolentzos et al. (2017). In Donnat et al. (2018a) authors analyze graph-based distance methods for a temporal graph of bio-medical surveys.

Hierarchical clustering and fusion of different network representations were overviewed in Yang and Wang (2018). Usually, this kind tasks require fusion of different similarity representations of a network as different graphs (Serra et al. (2015); Xue et al. (2015)), preserving graph structure (Hou et al. (2017) or simultaneously performing semi-supervised classification and clustering with adaptive kNN model Nie et al. (2017a)). Different domain network clustering was suggested in Cheng et al. (2013) and improved in the following works suggesting fusion of different not-synchronized networks with different structures ( $\mathrm{Ni}$ et al. (2016)), cross-domain associations (Liu et al. (2015b)) or multi-view spectral clustering (Li et al. (2015b)). Khasahmadi et al. (2020) propose a memory layer for graphs, that can efficiently learn graph hierarchical representations. Tsitsulin et al. (2020) propose an algorithm for efficient calculation of spectral distances for large graphs. Kolouri et al. (2020) suggest the embedding preserving Wasserstein distance with linear complexity. Qin et al. (2020) presents one more graph pooling technique that uniformly aggregates neighbourhood. Baldini et al. (2020) embeds maximal cliques to preserve structural similarities between graphs. Yan and Wang (2020) states the problem of transfer learning suggesting the framework for graph alignment and further adaptation learning for GNNs.

\subsection{Network visualization Definition 11 (Graph visualization) is a way to map a graph to a low $(2 D, 3 D)$ dimensional space.}

All nodes are either directly embedded as 2D vectors (Le and Lauw (2014); Wang et al. (2016); Cao et al. (2016); Tu et al. (2016); Niepert et al. (2016); Pan et al. (2016)) or first embedded to certain dimension, and then compressed via PCA (Herman et al. (2000)) or t-SNE (Maaten and Hinton (2008)) (or other dimension reduction frameworks, see for, e.g., Tenenbaum et al. (2000); De Oliveira and Levkowitz (2003)) in order to plot in 2D space. If there are labels or communities representative for network dataset, the nodes are usually visualized with different colours for each label in order to verify whether similar nodes are embedded closer to each other. Such models, as Perozzi et al. (2014); Grover and Leskovec (2016); Tang et al. (2015); Ou et al. (2016); Wang et al. (2016) demonstrated proper performance on the task of network visualization for unsupervised graph embedding models. Evaluation of graph embeddings for large structural data visualization can be found in Tang et al. (2016a). Graph visualization techniques beyond planar mappings can be found in Didimo et al. (2019).

\subsection{Network compression}

Definition 12 (Network compression, simplification or sparsification) is a task of reducing the number of nodes and edges in a graph, for further efficient application of graph algorithms.

The concept of network compression was first introduced in as Feder and Motwani (1991) under the idea of reducing the number of stored graph edges while achieving a faster performance of certain algorithms on graphs. The compression was made by grouping nodes and edges into partitions of bipartite cliques and then replacing these cliques with trees. Similar ideas of dividing the graph into groups of nodes and edges and encoding them were proposed in several studies Pardalos and Xue (1994); Tian et al. (2008); Toivonen et al. (2011). Minimum Description Length (MDL) (Rissanen (1978)) was used in Navlakha et al. (2008) to construct graph summary adjusted with edge correction algorithm.

Graph embeddings support compact graph representation, reducing memory storage from $O(|V| \times|V|)$ to $O(d \times|V|)$, where embedding dimension $d \ll n$ below 200 was shown to be enough for qualitative network reconstruction for second-order preserving proximity models (e.g. link prediction), such as $\mathrm{Ou}$ et al. (2016) and Wang et al. (2016). They also suit for various graph optimization task providing useful tools for constructing graph-based heuristics (Khalil et al. (2017)). 


\section{APPLICATIONS TO REAL-WORLD PROBLEMS}

In this section, we are interested in how graph embeddings appear in many other computer science fields, in which graphs are not directly expressed in the data, but relations between the objects can be efficiently described by graphs, and so, graph embeddings.

\subsection{Computer vision}

Image classification can be solved with classic $\mathrm{CNN}$ models considering the images as a grid-like structure. Recently, graph convolutional network models can take into account different neighbouring relations, thus going beyond the nearest pixels as the only features for convolutions. Especially interesting results were obtained for 3D shape reconstruction (Monti et al. (2017a)) and video action recognition.

There are four main ideas of using graph neural networks for computer vision tasks: working with the interaction of objects on video and images, feature similarity graph, label graph, i.e. images with the same label are connected, and internal graph-structured image data.

One of the main problems with CNN is that they should be deep enough to account interaction information between object, so Chen et al. (2019c) propose GloRe unit that applies GCNs over interaction data. It helps to efficiently solve relational reasoning task. In Wang et al. (2018) relation graph of image objects was built for localizing object instance from natural language expression. Graph representation is also useful for representing in-label object interaction like in metric learning. It successfully applied to face clustering task (Yang et al. (2019), Wang et al. (2019b)). Also such graph was exploited by Kim et al. (2019) for few-shot learning classification. Graph Convolutions are widely used in skeleton-based action recognition. It applies different graph network models to human skeleton graph (Shi et al. (2019), Si et al. (2019), Li et al. (2019a)). GNNs are used for video tracking and classification tasks Zhang et al. (2018a), Gao et al. (2019), Zhong et al. (2019).

\subsection{Natural language processing}

NLP is highly correlated to graph tasks. Here similar sequential methods are used, while data have hierarchical structure from different views.

In Marcheggiani and Titov (2017), authors assign semantic roles by encoding sentences with the graph convolutional network. In Marcheggiani et al. (2018), Zhao et al. (2019) graph convolutional network models were applied for machine translation. Sevgili et al. (2019) use the Wikipedia link graph between entities to improve the quality of entity disambiguation task on unstructured text data. Graph models are widely used in NLP to extract syntactic and semantic information (Luo et al. (2019); Vashishth et al. (2019); Veyseh et al. (2019)). The main approach is to extract the dependency graph and learn node (word) embeddings using GCN. Another approach is to examine each sentence as a complete graph with adjacency weighted by attention.

Graph neural networks also help in sequence tagging task, because it natively exploits information about the connection between different entities. Zhu et al. (2019) propose the Generated Parameters GNN for the Relation extraction task. It also builds a complete graph of entities in the sentence via encoding of the sentence with any sequence model. After that, GNN is applied to solve the node classification task. A prominent application of GNNs is to encode dependency tree information. Such an approach is exploited by Guo et al. (2019), they apply Graph Attention Models. Sahu et al. (2019) also use dependency graph for relation extraction tasks, but their model accounts for inter-sentence dependencies.

Question answering, comment generation and dialogue systems are highly dependent on domain knowledge-base. Such knowledge-base usually can be depicted as knowledge graphs. Banerjee and Khapra (2019); Kim et al. (2018a) applies GNN to encode knowledge and account to it in these tasks. Li et al. (2019b) also use graph models based on news interaction graphs.

The transformer-based language models (Vaswani et al. (2017)) works in a similar way to graph attention networks. It models a sentence as a complete graph and calculates new word representation weighting previous vectors with self-attention. The BERT model (Devlin et al. (2018)) is a special case of transformer-based models. It learns the vector by predicting masked words. Such tasks can be formulated as link prediction between context and masked words.

\subsection{Knowledge graph completion}

Knowledge graph embedding aims to learn vectors for entities and multi-dimensional vectors for entity relations. Knowledge graph completion solves link prediction between entities in knowledge graphs thus 
predicting ordered triples of entity-relation-entity (Lin et al. (2015)). Knowledge graph (KG) embedding presents a knowledge base as a collection of triples "head-relation-tail" and consider them training samples. Structured Embedding (Bordes et al. (2011)) learns two separate entity-relation representations for head and tail, while Semantic Matching Energy (Bordes et al. (2012)), Latent Factor Model (Jenatton et al. (2012)) and Neural Tensor Network (Socher et al. (2013)) embed entities and relations, and use models to capture correlations between them.

A survey on KG embeddings Wang et al. (2017a) considers translation-based models, such as TransE (Bordes et al. (2013)), TransH (Wang et al. (2014)), TransM (Fan et al. (2014)), TransR/CTransR (Lin et al. (2015)), TransC (Lv et al. (2018)), TransD (Ji et al. (2015)), TranSparse (Ji et al. (2016)), KG2E (He et al. (2015)), and semantic matching models, based on RESCAL (Nickel et al. (2011)) tensor factorization framework, such as DistMult (Yang et al. (2014)), HolE (Nickel et al. (2015)) and ComplEx (Trouillon et al. (2017)) with comparison paper for the latter two in Trouillon and Nickel (2017).

Question answering via knowledge graph embeddings was suggested in Huang et al. (2019). Weighted attention for supporting triple in KG link prediction problem was presented in Mai et al. (2018).

\subsection{Data mining}

Ye et al. (2019) proposed method that models relations between different entities in Android logs (API, apps, device, signature, affiliation) using a hierarchical graph. Then they classify nodes of such graphs for real-time malware classification. Graph neural networks are widely used to utilize the social network information. Wu et al. (2019a); Song et al. (2019); Chen et al. (2019a) use such models to account for social effects in recommender systems. Zhang et al. (2019a) propose Graph HyperNetworks for neural architecture search. It learns topology of architecture and infers weights for it.

\subsection{Recommender systems}

The basic approach for recommending top $\mathrm{K}$ nodes of interest for a given node is usually based on certain similarity metric (Pirotte et al. (2007); Zhao et al. (2013); Gui et al. (2016); Zhou et al. (2017); Ou et al. (2016)). There are various situations in which one need to provide node recommender system Zhang and Wang (2016), in particular, for items to customers via APP model (Zhou et al. (2017)), documents matching a given query (Xiong et al. (2017)), community-based question answering (Zhao et al. (2016); Fang et al. (2016)), music recommendations via user preference embedding for query answering (Chen et al. (2015a, 2016a)), location recommendations (Xie et al. (2016)), and many other real-world scenarios.

Matrix completion approach based on graph embeddings was provided in Monti et al. (2017b). Large scale recommender system was presented in Ying et al. (2018a). Explainable recommendations were studied in Zhang and Chen (2018). In Zhang et al. (2019d) authors represents product search as a graph of co-clicked answers. They mix network embedding, term item vectors and term query vector using MLP to predict the probability of click on the item in certain query. This score is used to rank products.

STAR-GCN (Zhang et al. (2019c)) is used over user-item interaction graph to learn user and item vectors. This approach is also suitable for inductive learning only using several interactions of users and items. This helps to solve the cold-start problem in recommender systems. Shang et al. (2019) use graphs for encoding hierarchical structure of health diseases. Next, achieved embeddings are integrated into BERT model for visit-based user recommendation.

The classical specific case of using network science in recommendations is the link prediction in collaborator networks (Chen et al. (2005); Liu and Kou (2007); Li and Chen (2009); Cho and Yu (2018)). Kong et al. (2018) developed a scientific paper recommender system based on citation networks, which uses text information embeddings to find papers of similar research interest and structural network embedding. The combined embedding model was then applied for constructing article vector representations. A combination of network and knowledge graphs was proposed in Yang et al. (2016b). In Makarov et al. (2019a,b,c) authors show that two-level architecture can improve the recommendation results. Firstly it predicts the collaboration itself and further estimates its quantity/quality. A survey on co-authorship and citation recommender systems may be found in Ortega et al. (2018b).

\subsection{Biomedical data science}

The large variety of data in biomedicine can be represented as networks. Le et al. (2019) applies embedding techniques to electron transport chains. Do et al. (2020) utilizes it for detection of specific proteins. Lin et al. (2020b) exploits the dynamic graph embedding for detecting changes in functional connectivity in the brain network. 
Computational drug design is an attractive direction because it reduces the costs of development of new drugs. The prominent field is drug repositioning. It usually works with networks of drug interaction with other entities: target, disease, gene or another drug. The main idea of such task is to predict possible relations between drug and other entities Su et al. (2020). For example, drug-disease interaction networks can predict the possible treatment of new disease with existing drugs. So, it is a similar statement to the link prediction problem. Yamanishi et al. (2008); Cobanoglu et al. (2013); Ezzat et al. (2017) find drug-target pairs via proximity over matrix factorization based embeddings. Zheng et al. (2013); Yamanishi et al. (2014); Ezzat et al. (2016) try to add external data to the drug-interaction network embeddings. Luo et al. (2017); Zong et al. (2017); Alshahrani et al. (2017) build heterogeneous networks of different drug-related interaction and apply network embedding methods to it. Wang et al. (2019a) embeds heterogeneous gene graph to predict drug response.

Another important field in medicine design is the adverse drug reaction (ADR) analysis. Some papers Zitnik and Zupan (2016); Zitnik et al. (2018) focus on similar drug-drug and drug-target interaction prediction. Wang (2017); Abdelaziz et al. (2017) use the knowledge graph based on biomedical texts. Stanovsky et al. (2017) also works with KG embedding, but over ADRs mentions in social media.

Network science is also applied to the molecule structure. Li et al. (2017b) proposes a prediction of pathogenic human genes using network embedding. Network embedding is very popular method in protein-protein interaction assessment and function prediction (Kulmanov et al. (2018); Su et al. (2020); Wang et al. (2017b)). Shen et al. (2017); Li et al. (2017a) applies to miRNA-disease interaction network to associate genes with complex diseases. The detailed survey of biomedical network embedding applications is presented by Su et al. (2020).

\subsection{Reinforcement learning}

Reinforcement learning (RL) is a popular approach to solve combinatorial optimization problems. Zheng et al. (2020b) provides the open-sourced environment for graph optimization problems using reinforcement learning and graph embeddings. Hayashi and Ohsaki (2020) use RL for a similar task, such as binary topology optimization of trusses. It utilizes graph convolution networks for feature extraction and further usage in RL optimization. A similar concept was used in Yan et al. (2020) to solve automatic embedding problem using actor-critic models for optimization and graph embeddings for representation learning.

Waradpande et al. (2020) suggests encoding states in Markov's decision process with graph embedding models.Lin et al. (2020a) follows this idea and utilizes GNN for parametrization of the stochastic policy in electric vehicle routing problem. Zhou et al. (2020) solves the interactive recommender system problem enhancing it with knowledge graphs. It describes states using GCN over knowledge graph.

\section{OPEN PROBLEMS}

Here we mention the most interesting open problems in graph representation theory, which are far from good results applicable for any given scenarios.

Many real-world graphs are dynamic: nodes and edges can appear and vanish over time. Despite a large number of recent papers, this field is far from benchmark well-performing models as of now. One of the approaches for it is inductive learning, which is strongly correlated with graph dynamics problem. Inductive methods allow finding embedding for newly added nodes without refitting the whole model. It is important in real-world applications and partially solve the scalability issue.

Edge attributes aware network embedding is poorly studied field. There is a low number of models. Such models usually depend on a Line graph, which has a dramatically larger number of nodes. So such models have a problem with scalability. Edge attributes are important in such tasks as context-aware recommender systems or transportation networks optimization.

They are an only little number of works about subgraph embedding. Such models should represent complex structures like triangles or hierarchy. The application of non-euclidean spaces to the embedding task is a promising method solving this issue, but also poorly studied.

Recent advances in the distributed and batch training for graph neural networks looks promising. However, most of the methods are not theoretically grounded, so it could be hard to understand the issues of poor quality of results. Only Zeng et al. (2019) provides some bias-variance analysis of node and edge sampling approaches. However, Akyildiz et al. (2020) provides a much faster and powerful method for large scale embedding. 
Another field that is currently under the control of many papers is the heterogeneous graph embedding. Such graphs are very common in real-world scenarios. The graph attention-based methods look promising in that field. It allows us to catch different aggregation levels like in Fu et al. (2020); Li et al. (2020).

As can be seen from our survey, most embedding models catch specific graph attributes and there is no general model, thus, raising a problem of selection and recommendation of different models for specific use-cases.

It is also an interesting point to develop meta-strategies for embedding mixture, that will preserve different graph properties. Such meta-models could solve the problem of knowledge generalization and reduce costs for deploy of application.

As in the other fields like NLP and CV, graph neural networks are poorly interpretable, apart from an initial study in Ying et al. (2019).

These and many other research questions lead to a vast amount of open research directions, which will benefit the field and lead to many applications in other computer science domains.

In our study, we focus on another interesting question regarding the fact that there are almost no general studies that compare the performance of models based on graph properties, most of the models are created for specific graph use-case. Below, we provide our insights on real-world networks as well as interpretations on such findings.

\section{MODEL COMPARISON}

This paper focuses on the four most popular tasks on graphs: node classification, link prediction, node clustering and network visualization. These tasks cover most of the real-world applications, in which a graph is used to unify information on nodes and their properties.

\subsection{Data}

Table 2. Datasets description

\begin{tabular}{l|cccccc} 
& Assortativity & Label Modularity & \# Nodes & \# Edges & \# Features & \# Classes \\
\hline CORA & 0.7711 & 0.8061 & 2708 & 10556 & 1433 & 7 \\
CITESEER & 0.6754 & 0.8872 & 3327 & 9228 & 3703 & 6 \\
HSE & - & - & 4181 & 12004 & 0 & 0 \\
MAG CS & 0.7845 & 0.6989 & 18333 & 163788 & 6805 & 15
\end{tabular}

We use four benchmark datasets for comparison of different models: CORA (Sen et al. (2008)), Citeseer (Lim and Buntine (2016)), HSE coauthor network (Makarov et al. (2018)), and Microsof Academic Graph Computer Science (MAG CS) (Sinha et al. (2015)).

First two datasets are citation networks. This type of networks is very common for evaluating the quality of network embeddings. Also, these datasets are convenient for comparison of models, because they have interesting label and feature structure. The last dataset is a co-authorship network. It has heterogeneous edges and large size. General puspose graph embedding models work only with homogeneous graphs, so we merge all the edges between nodes in one edge. A brief overview of the datasets statistics is provided in Table 2.

\subsection{Metrics}

We use standard classification metrics for node classification and link prediction tasks.

- Accuracy is the rate of the right answer of a classifier.

- Precision is the rate of true positive answers relative to the number of all positive answers of a classifier.

- Recall is the rate of true positive answers relative to the number of all positive examples in the data.

- F1 is a harmonic mean of precision and recall.

- Area Under ROC-curve shows the probability that a random negative example sampled from a uniform distribution is ranked lower than randomly sampled positive. 
- Average precision is the average of all possible precision values weighted by the recall for different probability thresholds.

We calculate the standard deviation with the following procedure:

1. Generate subsample of data with $90 \%$ volume of a given dataset.

2. Train model on it

3. Estimate quality of the trained model on the test set.

4. Repeat previous steps nine more times.

Described bootstrap (Efron (1992)) procedure allows to easily calculate standard error and confidence intervals for any statistics. Confidence intervals are required to understand the significance of the difference between models.

Node clustering was evaluated with two metrics: silhouette coefficient and modularity.

- Silhouette score shows the average similarity between each example and its cluster in comparison with the closest another cluster, so it measures the overall cluster separation relative to the distance measure. In our study, we use the Euclidean distance.

- Modularity score works pretty similarly but for computing inter- and intra- cluster quality, it measures the density of connections between clusters, respectively to its density inside clusters.

We also evaluate the quality of node clustering and network visualization with a visual comparison of how clusters are grouped in the UMAP (McInnes et al. (2018)) projection of embeddings. UMAP (Uniform Manifold Approximation and Projection) is the dimensionality reduction technique based on topology and Riemannian geometry. Firstly, it builds the weighted nearest neighbours graph according to elements feature vectors (embeddings). Then, it initializes layout using spectral embedding and optimizes it using SGD minimizing fuzzy-set cross-entropy.

The UMAP works much faster then TSNE and gives at least the same quality of the projection. Interpretation of received plot is simple: similar samples in initial space (for e.g., nodes with the same labels) should lie closely in the 2D plane.

\subsection{Evaluation pipeline}

The node classification task is native multi-class classification. Link prediction task can be also solved as classification, but with two classes depicting edge existence. The basic approach on validating such methods is to use delayed sample. So, before any model was trained, we create a train-test split for all the datasets, in order to compare all the models on similar subsets. We use simple 50\% test, $50 \%$ train random split for node classification, following other papers on graph embeddings.

The problem with link prediction is that described graphs are high-imbalanced because there are much more unconnected node pairs. Large imbalance leads to poor training because even simple constant prediction will give high-scores. One of the methods for working with this problem is to the under-sample larger class. To keep the classification task harder, it is convenient to use a negative sampling technique. We select the most similar pairs of nodes which are not connected in the same amount as existent edges. The used proximity metric is cosine similarity, which is a normalized dot product of feature vectors. For features, we use the adjacency matrix.

Because basic classification models do not work with discrete graph data, after developing train and test samples, we need to generate meaningful features. Here we use the unsupervised graph embeddings (128 dimensions as commonly used in different papers and surveys). Graph neural networks were also trained in an unsupervised way with reconstruction loss over the graph adjacency matrix. Reconstruction loss calculates with binary cross-entropy between adjacency matrix and its estimation achieved by inner-product decoding from the embedding matrix.

Now, we can solve downstream tasks like classification or clustering. For that part, we use three different classifiers: logistic regression (LR), random forest (RF) and gradient boosting (GBM). Logistic regression is a linear model: it calculates the weighted average of object features and normalizes it with sigmoid to receive probability. Linear models are fast, interpretable and easily tunable because of their 
simplicity. Random forest is the ensemble of decision trees built on bootstrapped subsamples in both dimensions features and observations. Gradient boosted machines are another approach to learn decision tree ensemble. It determines each next tree by sequential optimization of the previous learner error-term. The main advantage of the tree-based models is that they could recover non-linear dependencies. But for this flexibility, we pay with a strong dependence on hyperparameter selection. Scikit-learn implementation with default hyperparameters was used. In the link prediction, we simply concatenate node vectors to achieve edge embedding. For the clustering, we use the $K$-means model from Scikit-learn.

Remark. The common way to use graph neural networks is semi-supervised training. Such an approach gives a bias towards the usage of that model, because embedding learns not only graph structure and feature transformations, but supervised information about labels on the other hand. So we train graph neural networks in an unsupervised way because our study is aimed to understand how different properties of embedding models can help in considered tasks.

\subsection{Models}

We select several models of different types mentioned in Section 3 that preserve different properties of a graph. The general idea is to compare models of different fitting approaches with respect to network properties.

Matrix factorization based models:

- GraRep is symmetric and preserves high-proximity. The default $K$-hop order is 5 , the number of SVD iterations is 20 , a random seed is 42 .

- HOPE directly models asymmetric similarities.

- M-NMF preserves community structure. The default number of clusters is 20 , clustering penalty is 0.05 , modularity regularization penalty is 0.05 , similarity mixing parameter is 5 , the number of power-iterations is 200 , early stopping step is 3 .

Random-walks based models:

- Node2vec is a baseline for sequential methods which efficiently trade-offs between different proximity levels. The default walk length is 80 , the number of walks per node is 10 , return hyper-parameter is 1 , in-out hyper-parameter is 1 .

- Diff2vec use diffusion to sample random walks. The default number of nodes per diffusion tree is 80 , the number of diffusions per source node is 10 , context-size is 10 , the number of ASGD iterations is 1 , the learning rate is 0.025 .

- Walklets allow to model different levels of community structure and generalize GraRep model. Default random walk length is 80 , the number of random walks per source node is 5 , the window size is 5 , the minimal number of appearances is 1 , the order of random walk is first, return hyper-parameter is 1 , in-out hyper-parameter is 1 .

- GEMSEC directly cluster nodes. Default random walk length is 80 , the number of random walks per source node is 5 , the window size is 5 , the minimal number of appearances is 1 , the order of random walk is first, return hyper-parameter is 1 , in-out hyper-parameter is 1 , distortion is 0.75 , negative samples number is 10 , the initial learning rate is 0.001 , annealing factor for learning rate is 1 , initial clustering weight coefficient is 0.1 , final clustering weight coefficient is 0.5 , smoothness regularization penalty is 0.0625 , the number of clusters is 20 , normalized overlap weight regularization.

Deep learning models:

- GCN is a baseline for deep learning models. The default number of epochs is 200 , dropout is 0.3 , the learning rate is 0.01 , weight decay is 0.0005 , the number of hidden layers is 1 .

- GraphSage (GS) improves GCN by reducing the number of neighbours while weighting the node vectors. The dropout is 0.1 , aggregation is GCN, the number of epochs is 200 , the learning rate is 0.01 , weight decay is 0.0005 . 
Table 3. Results of model validation on node clustering task (both metrics lie between $(-1,1)$ and higher value means better results) number of hidden layers is 1 .

\subsection{Results}

The current section has the following structure. We start the analysis from node clustering tasks because also helps to understand the performance of graph embeddings on the other tasks. Further, we describe de classification task and link prediction followed by network visualization. We also conducted experiments on random graphs to study the difference of graph embeddings on real-world networks and simulated ones.

\begin{tabular}{|c|c|c|c|c|c|c|}
\hline & \multicolumn{2}{|c|}{ CORA } & \multicolumn{2}{|c|}{ CITESEER } & \multicolumn{2}{|c|}{ HSE } \\
\hline & Modularity & Silhouette & Modularity & Silhouette & Modularity & Silhouette \\
\hline GRAREP Cao et al. (2015) & 0.2249 & 0.1902 & 0.0320 & 0.3159 & 0.2320 & 0.3163 \\
\hline HOPE Ou et al. (2016) & 0.1222 & 0.2593 & 0.1748 & 0.5492 & 0.0027 & 0.6684 \\
\hline M-NMF Wang et al. (2017d) & 0.0209 & 0.0885 & 0.0209 & 0.0608 & 0.0244 & 0.0464 \\
\hline NODE2VEC Grover and Leskovec (2016) & 0.0106 & 0.1000 & -0.0018 & 0.0464 & 0.0419 & 0.5576 \\
\hline DIFF2VEC Rozemberczki and Sarkar (2018) & 0.1289 & 0.5412 & 0.0292 & 0.5422 & 0.1155 & 0.5429 \\
\hline GEMSEC Rozemberczki et al. (2018) & 0.7684 & 0.2280 & 0.7555 & 0.1508 & 0.7710 & 0.1678 \\
\hline WALKLETS Perozzi et al. (2016) & 0.7353 & 0.0812 & 0.7263 & 0.0566 & 0.7593 & 0.0667 \\
\hline "GCN Kipf and Welling (2016a) & 0.3800 & 0.3336 & 0.3754 & 0.4215 & - & - \\
\hline GRAPHSAGE Hamilton et al. (2017a) & 0.6455 & 0.3311 & 0.5774 & 0.4757 & - & - \\
\hline GAT Veličković et al. (2017) & 0.7209 & 0.3477 & 0.7367 & 0.3797 & - & - \\
\hline 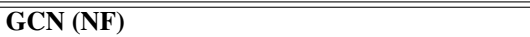 & -0.0096 & 0.3979 & 0.0360 & 0.4999 & 0.0008 & 0.6837 \\
\hline GRAPHSAGE (NF) & 0.0212 & 0.7672 & 0.0960 & 0.9442 & 0.0552 & 0.8381 \\
\hline GAT (NF) & 0.1335 & 0.2001 & 0.2968 & 0.3641 & 0.1400 & 0.6390 \\
\hline
\end{tabular}

Table 4. Results of model validation on node clustering task for MAG-CS dataset (both metrics lie between $(-1,1)$ and higher value means better results)

\begin{tabular}{l|llllllll} 
& HOPE & NODE2VEC & GRAREP & WALKLETS & GCN & GAT & GCN (NF) & GAT (NF) \\
\hline Modularity & -0.0001 & -0.0037 & 0.0027 & 0.0025 & 0.3462 & 0.3446 & 0.0112 & 0.1951 \\
Silhouette & 0.6548 & 0.0771 & 0.2348 & 0.0441 & 0.2369 & -0.0261 & 0.4654 & 0.0411
\end{tabular}

- GAT utilizes an attention mechanism. The number of epochs is 200 , in-dropout is 0.1 , attention dropout is 0.1 , the learning rate is 0.005 , the negative slope is 0.2 , weight decay is 0.0005 , the

GAT (NF)

Silhete 10.658

Node clustering. The results on the node clustering task are presented in Table 3. Rows depict different models, which are grouped by model type: matrix factorization, random walks, graph neural networks with and without features. On the columns, we can see results on different datasets. For each dataset, we calculate two metrics: modularity and silhouette score. Highlighted results are the best.

In node clustering task, results are pretty obvious: the embeddings, which work with community structure, perform the best in terms of modularity. GEMSEC directly penalizes embeddings for low modularity score with $K$-Means objective, Walklets catches this information by accounting for several levels of node neighbourhood. Importance of such information could be proven by the comparatively high value of GraRep model, that works pretty similar to Walklets.

Graph neural networks with features give comparatively better results, meaning that node content helps to describe graph structure. GraphSAGE and GAT efficiently utilize the local structure of the network. The main difference is that GAT aggregates over the entire neighbourhood, but GraphSAGE aggregates only over a fix-sized sample.

In the case of MAG CS graph (Table 4) the best results show GAT and GCN. It means that in the case of large, heterogeneous graph features play a major role. Interesting, that GAT without features works much better than other structural models. It could refer to the attention, that selects only the most important neighbours in node embedding construction. It seems that the attention mechanism helps in this case to distinguish heterogeneous edge nature.

GNN models trained in unsupervised fashion give poor results because they highly rely on the features when constructing embeddings even for learning graph structure. 
Table 5. Results of model validation on node classification task (accuracy metric lies between $(0,1)$ and higher value means better results)

\begin{tabular}{l|lll} 
CORA & & & \\
\hline & GBM & LR & RF \\
\hline GRAREP Cao et al. (2015) & $0.7610 \pm 0.0434$ & $0.7503 \pm 0.0323$ & $0.7751 \pm 0.0254$ \\
HOPE Ou et al. (2016) & $0.7518 \pm 0.0333$ & $0.3024 \pm 0.0308$ & $0.7614 \pm 0.0289$ \\
M-NMF Wang et al. (2017d) & $0.2596 \pm 0.0250$ & $0.2799 \pm 0.0324$ & $0.2633 \pm 0.0239$ \\
\hline NODE2VEC Grover and Leskovec (2016) & $0.2522 \pm 0.0200$ & $0.2441 \pm 0.0273$ & $0.2441 \pm 0.0257$ \\
DIFF2VEC Rozemberczki and Sarkar (2018) & $0.2212 \pm 0.0635$ & $0.2843 \pm 0.0387$ & $0.2500 \pm 0.0293$ \\
GEMSEC Rozemberczki et al. (2018) & $0.8338 \pm 0.0326$ & $0.8153 \pm 0.0390$ & $\mathbf{0 . 8 6 3 4} \pm \mathbf{0 . 0 2 5 1}$ \\
WALKLETS Perozzi et al. (2016) & $0.8142 \pm 0.0252$ & $0.8124 \pm 0.0317$ & $0.8327 \pm 0.0326$ \\
\hline GCN Kipf and Welling (2016a) & $0.7803 \pm 0.0378$ & $0.6588 \pm 0.0448$ & $0.7718 \pm 0.0380$ \\
GRAPHSAGE Hamilton et al. (2017a) & $0.8083 \pm 0.0358$ & $0.7385 \pm 0.0391$ & $0.8168 \pm 0.0316$ \\
GAT Veličković et al. (2017) & $0.8194 \pm 0.0304$ & $0.7455 \pm 0.0420$ & $0.8264 \pm 0.0324$ \\
\hline GCN (NF) & $0.3021 \pm 0.0204$ & $0.2969 \pm 0.0238$ & $0.2888 \pm 0.0194$ \\
GRAPHSAGE (NF) & $0.3017 \pm 0.0298$ & $0.3021 \pm 0.0305$ & $0.3017 \pm 0.0298$ \\
GAT (NF) & $0.3021 \pm 0.0305$ & $0.3021 \pm 0.0305$ & $0.3021 \pm 0.0305$ \\
& & & \\
CITESEER & & & \\
\hline & & & \\
GRAREP Cao et al. (2015) & GBM & & \\
HOPE Ou et al. (2016) & $0.5582 \pm 0.0577$ & $0.5110 \pm 0.0443$ & $0.5834 \pm 0.0453$ \\
M-NMF Wang et al. (2017d) & $0.5468 \pm 0.0346$ & $0.2663 \pm 0.0443$ & $0.5489 \pm 0.0378$ \\
\hline NODE2VEC Grover and Leskovec (2016) & $0.1767 \pm 0.0220$ & $0.1978 \pm 0.0241$ & $0.1909 \pm 0.0311$ \\
DIFF2VEC Rozemberczki and Sarkar (2018) & $0.1815 \pm 0.0253$ & $0.1806 \pm 0.0165$ & $0.1867 \pm 0.0237$ \\
GEMSEC Rozemberczki et al. (2018) & $0.2035 \pm 0.0373$ & $0.2239 \pm 0.0281$ & $0.1930 \pm 0.0287$ \\
WALKLETS Perozzi et al. (2016) & $0.6754 \pm 0.0343$ & $0.5867 \pm 0.0427$ & $\mathbf{0 . 7 1 7 5} \pm \mathbf{0 . 0 2 4 7}$ \\
\hline GCN Kipf and Welling (2016a) & $0.6291 \pm 0.0280$ & $0.6243 \pm 0.0228$ & $0.6480 \pm 0.0277$ \\
GRAPHSAGE Hamilton et al. (2017a) & $0.6312 \pm 0.0210$ & $0.5092 \pm 0.0272$ & $0.6342 \pm 0.0209$ \\
GAT Veličković et al. (2017) & $0.6450 \pm 0.0228$ & $0.5425 \pm 0.0192$ & $0.6586 \pm 0.0309$ \\
\hline GCN (NF) & $0.6733 \pm 0.0238$ & $0.5582 \pm 0.0443$ & $0.6763 \pm 0.0220$ \\
GRAPHSAGE (NF) & $0.2729 \pm 0.0272$ & $0.2317 \pm 0.0336$ & $0.2792 \pm 0.0260$ \\
GAT (NF) & $0.1996 \pm 0.0409$ & $0.1996 \pm 0.0409$ & $0.1996 \pm 0.0409$ \\
& $0.1996 \pm 0.0409$ & $0.1996 \pm 0.0409$ & $0.1996 \pm 0.0409$
\end{tabular}

Table 6. Results of model validation on node classification task for MAG-CS dataset (accuracy metric lies between $(0,1)$ and higher value means better results)

\begin{tabular}{l|lll} 
& GBM & LR & RF \\
\hline GRAREP Cao et al. (2015) & $0.1915 \pm 0.0162$ & $0.1404 \pm 0.0217$ & $0.1737 \pm 0.0169$ \\
HOPE Ou et al. (2016) & $0.1985 \pm 0.0233$ & $0.2255 \pm 0.021$ & $0.1665 \pm 0.0184$ \\
\hline NODE2VEC Grover and Leskovec (2016) & $0.1882 \pm 0.034$ & $0.2048 \pm 0.0332$ & $0.168 \pm 0.0195$ \\
WALKLETS Perozzi et al. (2016) & $0.1866 \pm 0.0171$ & $0.1527 \pm 0.0084$ & $0.1886 \pm 0.0189$ \\
\hline GCN Kipf and Welling (2016a) & $0.752 \pm 0.0177$ & $0.7317 \pm 0.0166$ & $\mathbf{0 . 7 5 6 8} \pm \mathbf{0 . 0 1 7 6}$ \\
GAT Veličković et al. (2017) & $0.6272 \pm 0.0188$ & $0.6424 \pm 0.0202$ & $0.6095 \pm 0.0208$ \\
\hline GCN (NF) & $0.2089 \pm 0.0154$ & $0.2255 \pm 0.021$ & $0.1738 \pm 0.0117$ \\
GAT (NF) & $0.2255 \pm 0.021$ & $0.2255 \pm 0.021$ & $0.2255 \pm 0.021$
\end{tabular}

The clustering results show that specific losses can dramatically increase quality on a specific task. As we will see further, such losses are also helpful in the node classification task preserving important graph properties.

Node classification. The results on node classification task are presented in Table 5. Rows show different types of models and columns show different datasets. For each dataset, we calculate accuracy for three different models: gradient boosted machines, logistic regression and random forest. Highlighted results are the best.

Models that have good performance in node clustering task also show high score in node classification. Labels in given datasets show different topics of articles, as soon as usually authors are dedicated to 
specific topics, so natural communities are constructed within these labels. This can also be proven by high modularity and assortativity coefficients of label communities for all graphs. In the classification task, it is also important to have a good separation of clusters, that could be measured by the silhouette coefficient. We can see those models that keep both high modularity and high silhouette work better.

Linear models show the comparatively lower score, but for random walk based embeddings, this difference is much less severe. Most of considered random walk models are based on Skip-Gram approach, which is a log-linear model. It reduces expression quality of the model but allows to learn vectors that perform well in linear models.

Results for MAG CS are presented in Table 6. Firstly, we compare fewer models, because we were not able to compute some embeddings for such a large graph, so we choose the fastest ones with good performance in other experiments. HOPE outperforms other classic non-attributed embeddings. It is also interesting that the linear mixture of embedding elements works much better for this embedding then others. One of the reasons for such behaviour is that in the large graphs, embeddings could be noisy and simple models could give better quality. It could be also a reason for the worse performance of $K$-hop based embeddings (Walklets and GraRep). But the main driver in node classification quality is the features of nodes. It could be seen from the high results of GCN and GAT models.

HOPE has a dramatic difference between linear and non-linear models because it estimates Katz centrality, which has non-linear nature. Also, we use HOPE implementation from GEM Goyal and Ferrara (2017), where node embedding is achieved as a concatenation of its self- and context- representations. The non-linear model helps to reconstruct the dot product decoder. A similar argument can explain diversity in neural network models, but it has less variance because of high clustering efficiency.

Link prediction. Table 7 shows results for link prediction task. It is separated into three groups by datasets. Rows represent different graph embedding models and columns show different second-level classification models: gradient boosted machines, logistic regression and random forest. Highlighted results are the best.

In the link prediction task, we can also see the importance of clustering. Links are more likely to occur within one community. The high-proximity models also work much better, because in that task we need to understand how similar are non-connected nodes.

The performance of the HOPE model in this task is more significant. HOPE model concentrates on preserving asymmetric transitivity. The older paper can not cite the newer one.

GCN without features performs much better than other graph neural networks. It accounts for the whole neighbourhood and directly uses the adjacency matrix to train embeddings.

Results for MAG CS (Table 8) are consistent with these findings. However, despite the good quality on the community clustering task, GAT without features shows pure performance on the Link prediction task. However, GCN without features is close to the GAT with features. It means that in this task it is necessary to account the whole neighbourhood.

A dramatic difference in the quality of linear and non-linear models can be explained by the objective of the link prediction task. It requires to model the proximity between to nodes. Such metrics are non-linear. So for reconstructing it from concatenated vectors of nodes, we need some non-linear transformations.

Graph visualization. We present results of node clustering jointly with network visualization using UMAP technique. The results for three different datasets are shown at Figure 1 for Cora, Figure 2 for Citeseer, and Figure 3 for HSE datasets, respectively.

The best visualization in terms of community structure seems to be Walklets and GraRep models, which give nicely distinguishable clusters in all the cases. Both models work in the same way with $k$-hop similarity of vertices. GEMSEC also provides separate cluster picture but creates a lot of noisy points.

Interestingly, that HOPE also split graphs into several parts, but we can see by the modularity score, such parts are not correlated with node communities. Such an effect could occur because HOPE embedding has non-homogeneous structure due to concatenation of self- and context- representations.

In the case of graph neural networks, except for GAT, all clusters have poor separation. Such effect occurs because GNN weights neighbourhood node attributes, so boundary nodes will be close. GAT allows mitigating this problem because utilizes the attention mechanism, which weights meaningless node neighbours to zero. 
Table 7. Results of model validation on link prediction task (accuracy metric lies between $(0,1)$ and higher value means better results)

\begin{tabular}{|c|c|c|c|}
\hline \multicolumn{4}{|l|}{ CORA } \\
\hline & GBM & LR & RF \\
\hline GRAREP Cao et al. (2015) & $0.8766 \pm 0.0056$ & $0.7585 \pm 0.0037$ & $0.9143 \pm 0.0021$ \\
\hline HOPE Ou et al. (2016) & $0.9422 \pm 0.0039$ & $0.6706 \pm 0.0032$ & $0.9478 \pm 0.0020$ \\
\hline M-NMF Wang et al. (2017d) & $0.6507 \pm 0.0038$ & $0.6252 \pm 0.0018$ & $0.6618 \pm 0.0022$ \\
\hline NODE2VEC Grover and Leskovec (2016) & $0.7047 \pm 0.0039$ & $0.6185 \pm 0.0037$ & $0.7060 \pm 0.0042$ \\
\hline DIFF2VEC Rozemberczki and Sarkar (2018) & $0.7780 \pm 0.0049$ & $0.7508 \pm 0.0045$ & $0.7413 \pm 0.0029$ \\
\hline GEMSEC Rozemberczki et al. (2018) & $0.9692 \pm \mathbf{0 . 0 0 3 0}$ & $0.6512 \pm 0.0052$ & $0.9653 \pm 0.0011$ \\
\hline WALKLETS Perozzi et al. (2016) & $0.9153 \pm 0.0058$ & $0.7073 \pm 0.0022$ & $0.9574 \pm 0.0017$ \\
\hline GCN Kipf and Welling (2016a) & $0.8784 \pm 0.0028$ & $0.7094 \pm 0.0041$ & $0.8978 \pm 0.0022$ \\
\hline GRAPHSAGE Hamilton et al. (2017a) & $0.8988 \pm 0.0050$ & $0.5668 \pm 0.0049$ & $0.9111 \pm 0.0028$ \\
\hline GAT Veličković et al. (2017) & $0.9127 \pm 0.0047$ & $0.5666 \pm 0.0063$ & $0.9337 \pm 0.0021$ \\
\hline GCN (NF) & $0.7852 \pm 0.0060$ & $0.7084 \pm 0.0033$ & $0.8014 \pm 0.0024$ \\
\hline GRAPHSAGE (NF) & $0.5459 \pm 0.0043$ & $0.5033 \pm 0.0021$ & $0.5459 \pm 0.0043$ \\
\hline GAT (NF) & $0.5033 \pm 0.0021$ & $0.5033 \pm 0.0021$ & $0.5033 \pm 0.0021$ \\
\hline \multicolumn{4}{|l|}{ CITESEER } \\
\hline & GBM & LR & RF \\
\hline GRAREP Cao et al. (2015) & $0.8786 \pm 0.0046$ & $0.7198 \pm 0.0049$ & $0.9254 \pm 0.0031$ \\
\hline HOPE Ou et al. (2016) & $0.8985 \pm 0.0074$ & $0.6358 \pm 0.0052$ & $0.9119 \pm 0.0029$ \\
\hline M-NMF Wang et al. (2017d) & $0.5926 \pm 0.0049$ & $0.5685 \pm 0.0033$ & $0.6215 \pm 0.0031$ \\
\hline NODE2VEC Grover and Leskovec (2016) & $0.6895 \pm 0.0050$ & $0.6315 \pm 0.0056$ & $0.6934 \pm 0.0046$ \\
\hline DIFF2VEC Rozemberczki and Sarkar (2018) & $0.7553 \pm 0.0038$ & $0.7258 \pm 0.0038$ & $0.7206 \pm 0.0060$ \\
\hline GEMSEC Rozemberczki et al. (2018) & $\mathbf{0 . 9 8 2 7} \pm \mathbf{0 . 0 0 3 1}$ & $0.6151 \pm 0.0096$ & $0.9726 \pm 0.0026$ \\
\hline WALKLETS Perozzi et al. (2016) & $0.8688 \pm 0.0066$ & $0.6672 \pm 0.0040$ & $0.9429 \pm 0.0024$ \\
\hline GCN Kipf and Welling (2016a) & $0.8863 \pm 0.0033$ & $0.6910 \pm 0.0032$ & $0.9052 \pm 0.0024$ \\
\hline GRAPHSAGE Hamilton et al. (2017a) & $0.8952 \pm 0.0037$ & $0.6082 \pm 0.0036$ & $0.8998 \pm 0.0034$ \\
\hline GAT Veličković et al. (2017) & $0.9175 \pm 0.0030$ & $0.6136 \pm 0.0051$ & $0.9306 \pm 0.0025$ \\
\hline GCN (NF) & $0.7892 \pm 0.0039$ & $0.6881 \pm 0.0044$ & $0.8100 \pm 0.0034$ \\
\hline GRAPHSAGE (NF) & $0.5181 \pm 0.0039$ & $0.5037 \pm 0.0026$ & $0.5181 \pm 0.0039$ \\
\hline GAT (NF) & $0.5037 \pm 0.0026$ & $0.5037 \pm 0.0026$ & $0.5037 \pm 0.0026$ \\
\hline \multicolumn{4}{|l|}{$H S E$} \\
\hline & GBM & LR & RF \\
\hline GRAREP Cao et al. (2015) & $0.9202 \pm 0.0068$ & $0.7956 \pm 0.0032$ & $0.9332 \pm 0.0022$ \\
\hline HOPE Ou et al. (2016) & $0.6590 \pm 0.0050$ & $0.6062 \pm 0.0055$ & $0.7022 \pm 0.0038$ \\
\hline M-NMF Wang et al. (2017d) & $0.6824 \pm 0.0058$ & $0.6277 \pm 0.0041$ & $0.7467 \pm 0.0032$ \\
\hline NODE2VEC Grover and Leskovec (2016) & $0.7257 \pm 0.0049$ & $0.6634 \pm 0.0034$ & $0.7592 \pm 0.0039$ \\
\hline DIFF2VEC Rozemberczki and Sarkar (2018) & $0.7850 \pm 0.0040$ & $0.7505 \pm 0.0037$ & $0.7795 \pm 0.0034$ \\
\hline GEMSEC Rozemberczki et al. (2018) & $\mathbf{0 . 9 7 2 4} \pm \mathbf{0 . 0 0 3 5}$ & $0.7065 \pm 0.0043$ & $0.9671 \pm 0.0013$ \\
\hline WALKLETS Perozzi et al. (2016) & $0.9484 \pm 0.0028$ & $0.7730 \pm 0.0035$ & $0.9615 \pm 0.0022$ \\
\hline GCN (NF) Kipf and Welling (2016a) & $0.8178 \pm 0.0021$ & $0.7867 \pm 0.0031$ & $0.8214 \pm 0.0030$ \\
\hline GRAPHSAGE (NF) Hamilton et al. (2017a) & $0.5071 \pm 0.0026$ & $0.5039 \pm 0.0030$ & $0.5071 \pm 0.0026$ \\
\hline GAT (NF) Veličković et al. (2017) & $0.5039 \pm 0.0030$ & $0.5039 \pm 0.0030$ & $0.5039 \pm 0.0030$ \\
\hline
\end{tabular}

It seems that one of the most important graph property in the studied tasks is the community structure. So, most of the methods, that works with it directly allow to achieve the best scores. It is also connected to the level of proximity because it is indirectly connected with the community structure. The graph neural networks allow to easily catch node attributes, but miss most of graph properties information, so it performs on the level of baseline models without it.

Random graphs. In order to understand robustness of graph embeddings, we decided to test how modelling real-world network with random graphs impact on the quality of graph embeddings for simulated networks.

Firstly, we should explain the formation of random graphs in different models. Erdős-Rényi (ER) 
Table 8. Results of model validation on link prediction task for MAG-CS dataset (accuracy metric lies between $(0,1)$ and higher value means better results)

\begin{tabular}{l|lll} 
& GBM & LR & RF \\
\hline GRAREP Cao et al. (2015) & $0.5986 \pm 0.0047$ & $0.5626 \pm 0.0016$ & $0.5998 \pm 0.0025$ \\
HOPE Ou et al. (2016) & $0.566 \pm 0.0017$ & $0.5275 \pm 0.0025$ & $0.6007 \pm 0.0027$ \\
NODE2VEC Grover and Leskovec (2016) & $0.578 \pm 0.0023$ & $0.5425 \pm 0.0015$ & $0.6137 \pm 0.0031$ \\
WALKLETS Perozzi et al. (2016) & $0.5798 \pm 0.0024$ & $0.5647 \pm 0.0015$ & $0.6077 \pm 0.0027$ \\
GCN Kipf and Welling (2016a) & $0.8486 \pm 0.0022$ & $0.6553 \pm 0.0014$ & $\mathbf{0 . 8 7 7 2} \pm \mathbf{0 . 0 0 1 2}$ \\
GAT Veličković et al. (2017) & $0.7293 \pm 0.0041$ & $0.5632 \pm 0.0024$ & $0.7524 \pm 0.0027$ \\
GCN (NF) & $0.7253 \pm 0.0012$ & $0.697 \pm 0.0014$ & $0.7261 \pm 0.0017$ \\
GAT (NF) & $0.5015 \pm 0.0009$ & $0.5015 \pm 0.0009$ & $0.5015 \pm 0.0009$
\end{tabular}
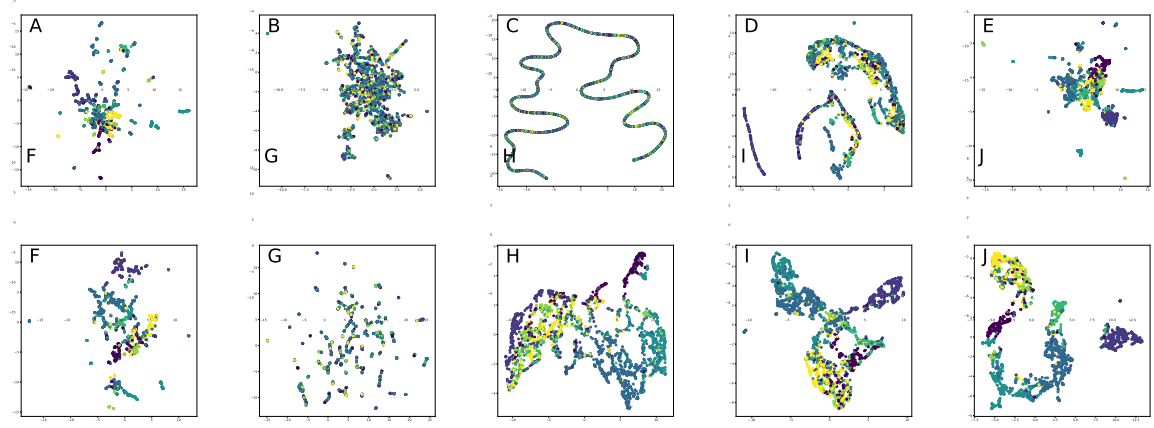

Figure 1. UMAP projection of CORA embeddings: (A) HOPE. (B) Node2Vec. (C) Diff2Vec. (D) GraRep. (E) Walklets. (F) GEMSEC. (G) M-NMF. (H) GCN. (I) GraphSage. (J) GAT.
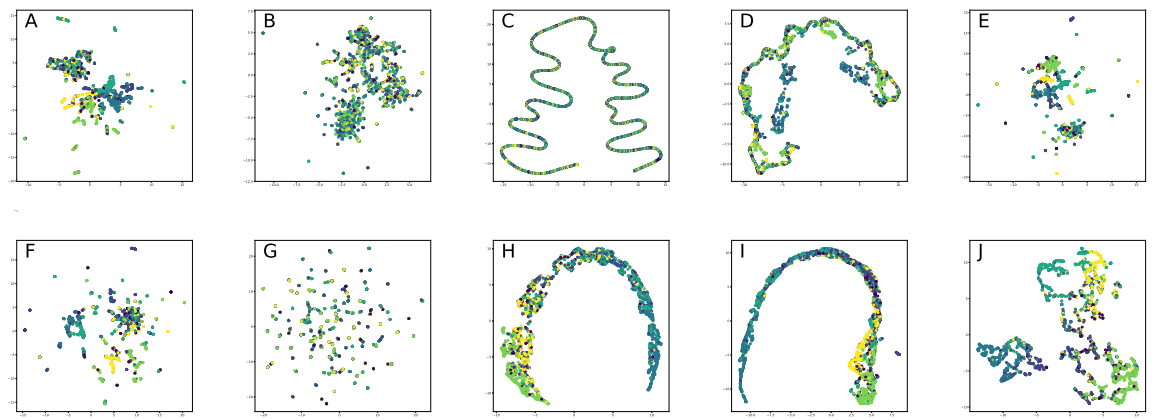

Figure 2. UMAP projection of Citeseer embeddings: (A) HOPE. (B) Node2Vec. (C) Diff2Vec. (D) GraRep. (E) Walklets. (F) GEMSEC. (G) M-NMF. (H) GCN. (I) GraphSage. (J) GAT.

builds the graphs using a simple binomial rule for creating an edge with a given density of graph (Erdös and Rényi (1959)). Barabási-Albert (BA) starts from a small graph and sequentially adds a new node with a given density and connects existing nodes using preferential attachment rule (Barabási and Albert (1999)). In the Watts-Strogatz (WS) model, the regular lattice is firstly constructed followed by edge rewiring procedure (Watts and Strogatz (1998)).

We build random graphs regarding the properties of real-world graphs. To build ER graph one need to have a number of nodes and edges in the graph. For the BA graph construction, it is required to have a number of nodes and number of edges for the newly added node at each iteration. It is a small integer, so we just select the best to fit the number of edges of benchmarks. The parameters of WS graphs were chosen based on the number of nodes, edges and average clustering of graphs following formulae: the number of edges in starting lattice is equal to $k=$ [2\# edges\# nodes], the rewriting probability is equal to $p=1-\sqrt[3]{4(k-1) 3(k-2)} \cdot($ average clustering) (Barrat and Weigt (2000)).

One of the main properties of all random graph models is the giant connected component. So 

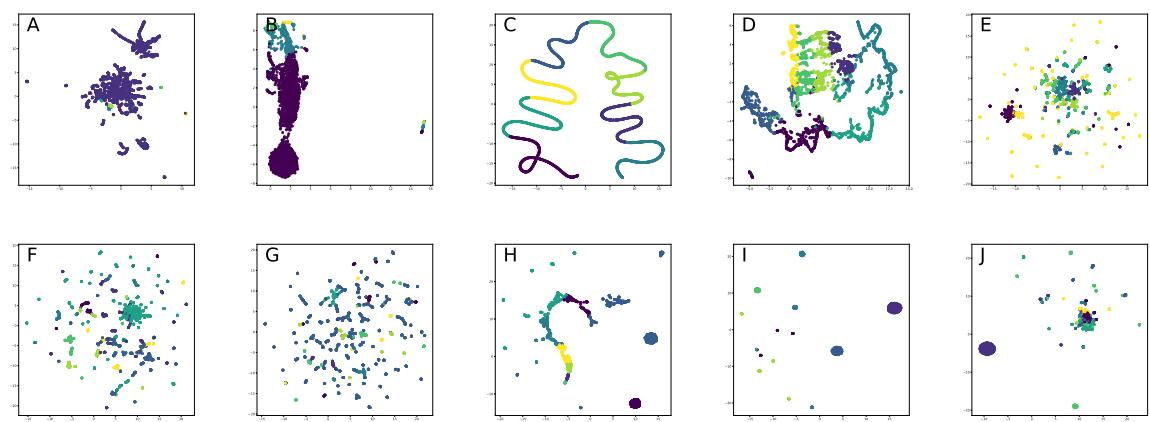

Figure 3. UMAP projection of HSE embeddings: (A) HOPE. (B) Node2Vec. (C) Diff2Vec. (D) GraRep. (E) Walklets. (F) GEMSEC. (G) M-NMF. (H) GCN. (I) GraphSage. (J) GAT.

embedding models learn it on the train part and works better than random in link prediction task in all cases. Additionally, in the BA model, there are some nodes with much larger density than others, so it is easier to predict missed links. Watts-Strogatz also has a straightforward mechanism of edge construction, where the shortest path is small. In both BA and WS models it is also possible to reproduce community structure. We can see it by large modularity metric in the clustering task.

Random graph modelling is one of the efficient methods in network science for evaluation of different model properties. For example, comparison of real-graph with its random analogue could help to understand how good is the received quality for the specific task. We follow this idea and compare two embeddings models Walklets and HOPE. We select these embeddings because it is non-context, show good performance and saves different properties. Walklets preserves $K$-hop similarity and HOPE preserves asymmetric transitivity.

Table 9. Results of model validation on node clustering task for random graphs (both metrics lie between $(-1,1)$ and higher value means better results)

\begin{tabular}{l|cccc} 
& \multicolumn{2}{|c}{ HOPE } & \multicolumn{2}{c}{ WALKLETS } \\
& Modularity & Silhouette & Modularity & Silhouette \\
\hline CORA (Original) & 0.1222 & 0.2593 & 0.7353 & 0.0812 \\
CORA (Barabási-Albert) & 0.0005 & $\mathbf{0 . 1 8 0 7}$ & 0.1465 & 0.0046 \\
CORA (Erdős-Rényi) & -0.0022 & 0.0216 & 0.0184 & 0.0080 \\
CORA (Watts-Strogatz) & 0.0629 & 0.1180 & $\mathbf{0 . 5 2 5 1}$ & 0.0212 \\
\hline CITESEER (Original) & 0.1748 & 0.5492 & 0.7263 & 0.0566 \\
CITESEER (Barabási-Albert) & -0.0008 & $\mathbf{0 . 3 7 3 1}$ & 0.0397 & -0.0006 \\
CITESEER (Erdős-Rényi) & 0.0031 & 0.1495 & -0.0040 & 0.0085 \\
CITESEER (Watts-Strogatz) & 0.0344 & 0.1270 & $\mathbf{0 . 4 9 4 1}$ & 0.0155
\end{tabular}

Similarly to the experiments on the real-world graphs, Walklets shows superior performance in comparison to the HOPE in Clustering (Table 9) and LPP (Table 10) tasks.

However, visually (Figure 4, 5) it better separates dense clusters. The Walklets visualization of random graphs differs from real-world cases. Random graphs give much sparser and visually harder to distinguish structure.

The results on random graphs and real networks differ sufficiently. It means that embedding models could really learn graph structure and its properties. Also, such citation networks are poorly described by random graph models.

\section{CONCLUSION}

In the current work, we present a comprehensive survey of graph embedding techniques. The work overviews different types of graph embeddings with respect to methods, network types, their applications to computer science domains.

One of the main achievements at the moment are the scalable models. The GNN could be trained in 
Table 10. Results of model validation on link prediction task for random graphs (accuracy metric lies between $(0,1)$ and higher value means better results)

\begin{tabular}{|c|c|c|c|}
\hline \multicolumn{4}{|l|}{ Walklets } \\
\hline & $\mathrm{RF}$ & LR & GBM \\
\hline CORA Original & $0.8142 \pm 0.0252$ & $0.8124 \pm 0.0317$ & $0.8327 \pm 0.0326$ \\
\hline CORA-ER & $0.617 \pm 0.0081$ & $0.5658 \pm 0.013$ & $0.5904 \pm 0.0141$ \\
\hline CORA-BA & $0.7216 \pm 0.0113$ & $0.6928 \pm 0.0103$ & $0.7271 \pm 0.0136$ \\
\hline CORA-WS & $0.6511 \pm 0.0329$ & $0.5168 \pm 0.0075$ & $\mathbf{0 . 7 4 4 2} \pm \mathbf{0 . 0 7 6 2}$ \\
\hline CITESEER Original & $0.6291 \pm 0.0280$ & $0.6243 \pm 0.0228$ & $0.6480 \pm 0.0277$ \\
\hline CITESEER-ER & $0.5505 \pm 0.0062$ & $0.5335 \pm 0.0071$ & $0.5411 \pm 0.0076$ \\
\hline CITESEER-BA & $0.6807 \pm 0.0071$ & $0.662 \pm 0.0123$ & $\mathbf{0 . 6 8 7 1} \pm \mathbf{0 . 0 1 8}$ \\
\hline CITESEER-WS & $0.571 \pm 0.0142$ & $0.5232 \pm 0.022$ & $0.6121 \pm 0.031$ \\
\hline \multicolumn{4}{|l|}{ HOPE } \\
\hline & $\mathrm{RF}$ & LR & GBM \\
\hline CORA Original & $0.7518 \pm 0.0333$ & $0.3024 \pm 0.0308$ & $0.7614 \pm 0.0289$ \\
\hline CORA-ER & $0.5936 \pm 0.0042$ & $0.5114 \pm 0.0055$ & $0.5734 \pm 0.0063$ \\
\hline CORA-BA & $\mathbf{0 . 6 5 2 1} \pm \mathbf{0 . 0 0 7 1}$ & $0.5559 \pm 0.0144$ & $0.6312 \pm 0.007$ \\
\hline CORA-WS & $0.5115 \pm 0.0048$ & $0.51 \pm 0.0052$ & $0.5132 \pm 0.0071$ \\
\hline CITESEER Original & $0.5468 \pm 0.0346$ & $0.2663 \pm 0.0443$ & $0.5489 \pm 0.0378$ \\
\hline CITESEER-ER & $0.5509 \pm 0.015$ & $0.5066 \pm 0.0029$ & $0.5439 \pm 0.01$ \\
\hline CITESEER-BA & $\mathbf{0 . 6 3 0 4} \pm \mathbf{0 . 0 1 1 6}$ & $0.5422 \pm 0.0071$ & $0.6096 \pm 0.0056$ \\
\hline CITESEER-WS & $0.5169 \pm 0.0057$ & $0.5093 \pm 0.0058$ & $0.521 \pm 0.0088$ \\
\hline
\end{tabular}
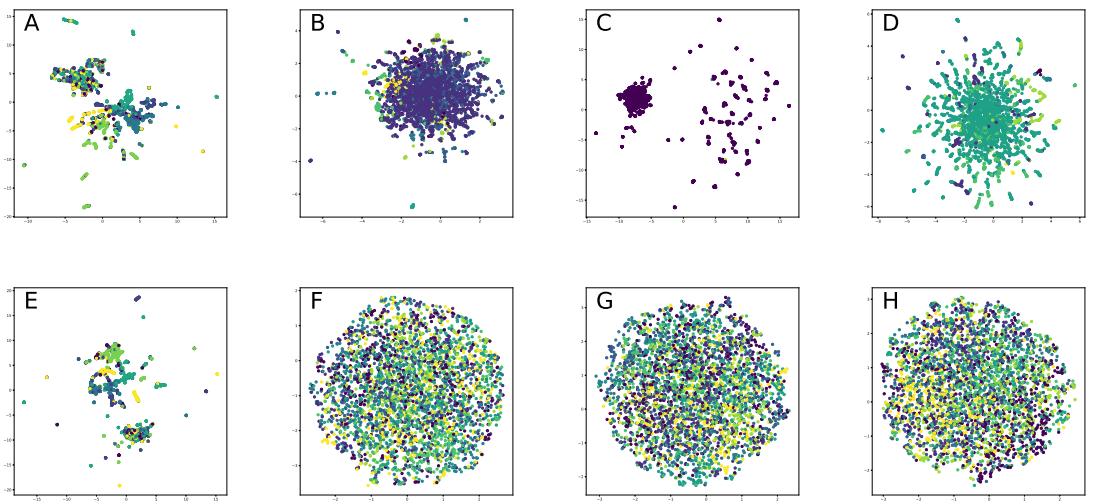

Figure 4. UMAP projection of Citeseer based random graph embeddings: (A) Original graph, HOPE. (B) Erdôs-Rényi, HOPE. (C) Barabási-Albert, HOPE. (D) Watts-Strogatz, HOPE. (E) Original graph, Walklets. (F) Erdős-Rényi, Walklets. (G) Barabási-Albert, Walklets. (H) Watts-Strogatz, Walklets.

batch and distributed fashion. Such methods allow using powerful attribute-aware models for real-world large graphs. However, only a few works analyze the proper strategies for batch sampling and its effect on the final results in terms of bias-variance trade-off. Another way to accelerate GNN training is to coarse a graph, but it could affect dramatically the final quality of the model. So, the understanding and further developing of coarsening and sampling techniques is the promising direction.

One of the most popular technique for graph embedding at the current time is the attention mechanism. It helps to account for some significant properties of a graph like temporality and heterogeneity by introducing attention in different dimensions: time, different levels of edge and nodes types. However, this method could be exhaustive in terms of computation, so it should be used with acceleration techniques.

The other research direction that grows rapidly is the stability of graph embeddings. The popular practices are to use variational graph autoencoders with Gaussian denoising or adversarial training.

The development of scalable and high-quality graph neural networks leads to an increase in the number 

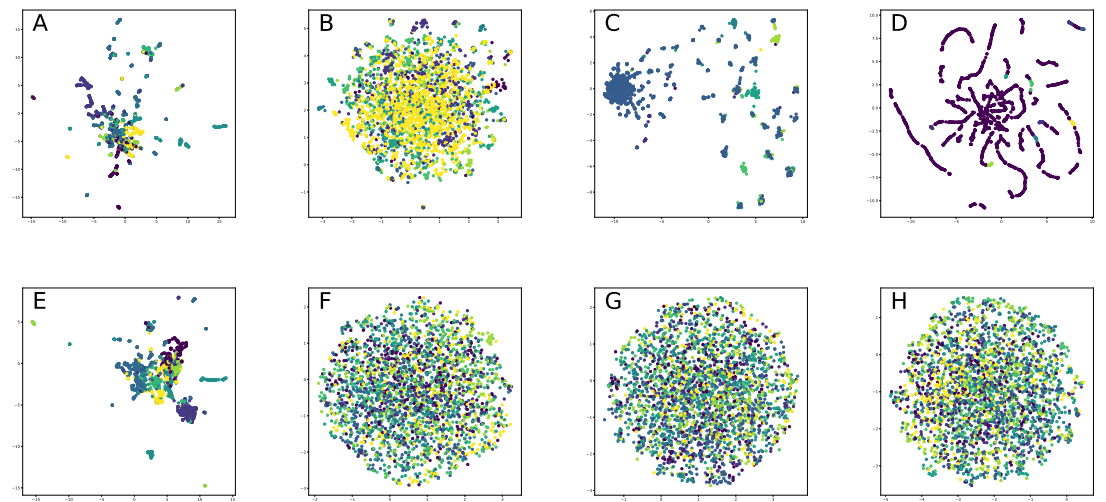

Figure 5. UMAP projection of CORA based random graph embeddings: (A) Original graph, HOPE. (B) Erdős-Rényi, HOPE. (C) Barabási-Albert, HOPE. (D) Watts-Strogatz, HOPE. (E) Original graph, Walklets. (F) Erdős-Rényi, Walklets. (G) Barabási-Albert, Walklets. (H) Watts-Strogatz, Walklets.

of applications to the non-graph domain. The most common application of it is the modelling of similarity or nearest neighbours graphs. Such approaches are presented in natural language processing, computer vision and recommender systems. However, in many fields, structures could be natively presented as graphs in terms of labels (samples of one type are connected), interaction, knowledge or relation graphs.

Our survey covers the most complete of methods and application in different computer science domains related to machine learning problems on relational data.

In addition, in the experiment part of the study we provide results on training best graph embedding models for node classification, link prediction, node clustering and network visualization tasks for different types of models and graphs to understand why certain graph embedding perform better than others on benchmark datasets under different training settings. Our experiments explain how different embeddings work with different properties uncovering graph inner properties and descriptive statistics impact on the models performance. As one of the most interesting findings, we show that structural embeddings with proper objectives achieve competitive quality versus graph neural networks.

Still, it could be hard to apply such methods to large graphs. Firstly, there is a problem with high computational complexity. Graph neural networks solve this issue by using batch training and sampling techniques. Another problem is that learned structural embeddings for large graphs could be noisy. However, adding the node attributes helps to concentrate on the specific important properties. Modern models focus on accounting for node attributes, but it was found that more important question is how to balance a trade-off between node attributes and network structure. Our work will be helpful in the further development of such generalization methods to answer this question. Such methods will allow to easily apply graph models in different domains.

\section{REFERENCES}

Abdelaziz, I., Fokoue, A., Hassanzadeh, O., Zhang, P., and Sadoghi, M. (2017). Large-scale structural and textual similarity-based mining of knowledge graph to predict drug-drug interactions. Journal of Web Semantics, 44:104-117.

Abu-El-Haija, S., Perozzi, B., and Al-Rfou, R. (2017). Learning edge representations via low-rank asymmetric projections. In Proceedings of the 2017 ACM on Conference on Information and Knowledge Management, pages 1787-1796. ACM, ACM.

Abu-El-Haija, S., Perozzi, B., Al-Rfou, R., and Alemi, A. A. (2018). Watch your step: Learning node embeddings via graph attention. In Advances in Neural Information Processing Systems, pages 9198-9208.

Adafre, S. F. and de Rijke, M. (2005). Discovering missing links in wikipedia. In Proceedings of the 3rd International Workshop on Link Discovery, LinkKDD '05, pages 90-97, New York, NY, USA. ACM. Adamic, L. A. and Adar, E. (2003). Friends and neighbors on the web. Social networks, 25(3):211-230. Ahmed, A., Shervashidze, N., Narayanamurthy, S., Josifovski, V., and Smola, A. J. (2013). Distributed 
large-scale natural graph factorization. In Proceedings of the 22nd international conference on World Wide Web, pages 37-48. ACM.

Akyildiz, T. A., Aljundi, A. A., and Kaya, K. (2020). Gosh: Embedding big graphs on small hardware. In 49th International Conference on Parallel Processing - ICPP, ICPP '20, New York, NY, USA. Association for Computing Machinery.

Alanis-Lobato, G., Mier, P., and Andrade-Navarro, M. A. (2016). Efficient embedding of complex networks to hyperbolic space via their laplacian. Scientific reports, 6:30108.

Alshahrani, M., Khan, M. A., Maddouri, O., Kinjo, A. R., Queralt-Rosinach, N., and Hoehndorf, R. (2017). Neuro-symbolic representation learning on biological knowledge graphs. Bioinformatics, 33(17):2723-2730.

as Feder, T. and Motwani, R. (1991). Clique partitions, graph compression, and speeding-up algorithms. In Proceedings of the 23rd Annual ACM Symposium on the Theory of Computing, pages 123-133. Citeseer.

Atahan Akyildiz, T., Alabsi Aljundi, A., and Kaya, K. (2020). Understanding coarsening for embedding large-scale graphs. arXiv e-prints, 2009.04925:arXiv-2009.

Atwood, J. and Towsley, D. (2016). Diffusion-convolutional neural networks. In Advances in Neural Information Processing Systems, pages 1993-2001.

Azran, A. (2007). The rendezvous algorithm: Multiclass semi-supervised learning with markov random walks. In Proceedings of the 24th international conference on Machine learning, pages 49-56. ACM.

Backstrom, L. and Leskovec, J. (2011). Supervised random walks: Predicting and recommending links in social networks. In Proceedings of the Fourth ACM International Conference on Web Search and Data Mining, WSDM '11, pages 635-644, New York, NY, USA. ACM.

Baldini, L., Martino, A., and Rizzi, A. (2020). Exploiting cliques for granular computing-based graph classification. In 2020 International Joint Conference on Neural Networks (IJCNN), pages 1-9. IEEE.

Baluja, S., Seth, R., Sivakumar, D., Jing, Y., Yagnik, J., Kumar, S., Ravichandran, D., and Aly, M. (2008). Video suggestion and discovery for youtube: taking random walks through the view graph. In Proceedings of the 17th international conference on World Wide Web, pages 895-904. ACM.

Banerjee, S. and Khapra, M. M. (2019). Graph convolutional network with sequential attention for goaloriented dialogue systems. Transactions of the Association for Computational Linguistics, 7:485-500.

Barabási, A.-L. and Albert, R. (1999). Emergence of scaling in random networks. science, 286(5439):509512.

Barrat, A. and Weigt, M. (2000). On the properties of small-world network models. The European Physical Journal B-Condensed Matter and Complex Systems, 13(3):547-560.

Belkin, M. and Niyogi, P. (2002). Laplacian eigenmaps and spectral techniques for embedding and clustering. In Advances in NIPS, pages 585-591. MIT press.

Berg, R. v. d., Kipf, T. N., and Welling, M. (2017). Graph convolutional matrix completion. arXiv preprint arXiv:1706.02263, 1706.02263.

Bhagat, S., Cormode, G., and Muthukrishnan, S. (2011). Node classification in social networks. In Social network data analytics, pages 115-148. Springer.

Bhagat, S., Cormode, G., and Rozenbaum, I. (2009). Applying link-based classification to label blogs. In Advances in Web Mining and Web Usage Analysis, pages 97-117. Springer.

Bojcheski, A. and Günnemann, S. (2018). Adversarial attacks on node embeddings. arXiv preprint arXiv:1809.01093, 1809.01093.

Bordes, A., Glorot, X., Weston, J., and Bengio, Y. (2012). Joint learning of words and meaning representations for open-text semantic parsing. In Artificial Intelligence and Statistics, pages 127-135. PMLR, MIT Press.

Bordes, A., Usunier, N., Garcia-Duran, A., Weston, J., and Yakhnenko, O. (2013). Translating embeddings for modeling multi-relational data. In Advances in neural information processing systems, pages 2787 2795. MIT press.

Bordes, A., Weston, J., Collobert, R., and Bengio, Y. (2011). Learning structured embeddings of knowledge bases. In Twenty-Fifth AAAI Conference on Artificial Intelligence, volume 25. AAAI.

Brand, M. (2003). Continuous nonlinear dimensionality reduction by kernel eigenmaps. In IJCAI, pages 547-554.

Brochier, R., Guille, A., and Velcin, J. (2019). Global vectors for node representations. arXiv preprint arXiv:1902.11004, 1902.11004. 
Bronstein, M. M., Bruna, J., LeCun, Y., Szlam, A., and Vandergheynst, P. (2017). Geometric deep learning: going beyond euclidean data. IEEE Signal Processing Magazine, 34(4):18-42.

Bruna, J., Zaremba, W., Szlam, A., and LeCun, Y. (2013). Spectral networks and locally connected networks on graphs. arXiv preprint arXiv:1312.6203, 1312.6203.

Cai, H., Zheng, V. W., and Chang, K. C.-C. (2017). A comprehensive survey of graph embedding: Problems. Techniques and Applications. arXiv preprint, 1709.07604.

Cao, M., Ma, X., Zhu, K., Xu, M., and Wang, C. (2020). Heterogeneous information network embedding with convolutional graph attention networks. In 2020 International Joint Conference on Neural Networks (IJCNN), pages 1-8. IEEE.

Cao, S., Lu, W., and Xu, Q. (2015). Grarep: Learning graph representations with global structural information. In Proceedings of the 24th ACM International on Conference on Information and Knowledge Management, pages 891-900. ACM.

Cao, S., Lu, W., and Xu, Q. (2016). Deep neural networks for learning graph representations. In AAAI, pages $1145-1152$.

Cavallari, S., Zheng, V. W., Cai, H., Chang, K. C.-C., and Cambria, E. (2017). Learning community embedding with community detection and node embedding on graphs. In Proceedings of the 2017 ACM on Conference on Information and Knowledge Management, pages 377-386. ACM.

Chamberlain, B. P., Clough, J., and Deisenroth, M. P. (2017). Neural embeddings of graphs in hyperbolic space. arXiv preprint arXiv:1705.10359, 1705.10359.

Chang, J. and Blei, D. (2009). Relational topic models for document networks. In Artificial Intelligence and Statistics, pages 81-88.

Chang, S., Han, W., Tang, J., Qi, G.-J., Aggarwal, C. C., and Huang, T. S. (2015). Heterogeneous network embedding via deep architectures. In Proceedings of the 21th ACM SIGKDD International Conference on Knowledge Discovery and Data Mining, pages 119-128. ACM.

Chen, C., Min, Z., Liu, Y., and Ma, S. (2019a). Social attentional memory network: Modeling aspectand friend-level differences in recommendation. In Proceedings of the Twelfth ACM International Conference on Web Search and Data Mining, pages 177-185, New York, NY, USA. ACM.

Chen, C.-M., Chien, P.-C., Lin, Y.-C., Tsai, M.-F., and Yang, Y.-H. (2015a). Exploiting latent social listening representations for music recommendations. In Proc Ninth ACM Int. Conf. Recommender Syst. Poster, pages 1-2.

Chen, C.-M., Tsai, M.-F., Lin, Y.-C., and Yang, Y.-H. (2016a). Query-based music recommendations via preference embedding. In Proceedings of the 10th ACM Conference on Recommender Systems, pages 79-82. ACM.

Chen, H., Anantharam, A. R., and Skiena, S. (2017a). Deepbrowse: Similarity-based browsing through large lists. In International Conference on Similarity Search and Applications, pages 300-314. Springer.

Chen, H. and Koga, H. (2019). Gl2vec: Graph embedding enriched by line graphs with edge features. In Gedeon, T., Wong, K. W., and Lee, M., editors, Neural Information Processing, pages 3-14, Cham. Springer International Publishing.

Chen, H., Li, X., and Huang, Z. (2005). Link prediction approach to collaborative filtering. In Proceedings of the 5th ACM/IEEE-CS Joint Conference on Digital Libraries (JCDL '05), pages 141-142, New York, NY, USA. ACM.

Chen, H., Perozzi, B., Al-Rfou, R., and Skiena, S. (2018a). A tutorial on network embeddings. arXiv preprint arXiv:1808.02590, 1808.02590.

Chen, H., Perozzi, B., Hu, Y., and Skiena, S. (2018b). Harp: Hierarchical representation learning for networks. In Thirty-Second AAAI Conference on Artificial Intelligence, pages 2121-2134.

Chen, J., Ma, T., and Xiao, C. (2018c). Fastgen: fast learning with graph convolutional networks via importance sampling. arXiv preprint arXiv:1801.10247, 1801.10247.

Chen, J., Shi, Z., Wu, Y., Xu, X., and Zheng, H. (2018d). Link prediction adversarial attack. arXiv preprint arXiv: $1810.01110,1810.01110$.

Chen, J., Wu, Y., Lin, X., and Xuan, Q. (2019b). Can adversarial network attack be defended? arXiv preprint arXiv:1903.05994, 1903.05994.

Chen, J., Wu, Y., Xu, X., Chen, Y., Zheng, H., and Xuan, Q. (2018e). Fast gradient attack on network embedding. arXiv preprint arXiv:1809.02797, 1809.02797.

Chen, J. and Zhang, A. (2020). Hgmf: Heterogeneous graph-based fusion for multimodal data with incompleteness. In Proceedings of the 26th ACM SIGKDD International Conference on Knowledge 
Discovery \& Data Mining, KDD ’20, page 1295-1305, New York, NY, USA. Association for Computing Machinery.

Chen, J., Zhang, Q., and Huang, X. (2016b). Incorporate group information to enhance network embedding. In Proceedings of the 25th ACM International on Conference on Information and Knowledge Management, pages 1901-1904. ACM.

Chen, J., Zhu, J., and Song, L. (2017b). Stochastic training of graph convolutional networks with variance reduction. arXiv preprint arXiv:1710.10568, 1710.10568 .

Chen, M., Tsang, I. W., Tan, M., and Cham, T. J. (2015b). A unified feature selection framework for graph embedding on high dimensional data. IEEE Transactions on Knowledge and Data Engineering, 27(6):1465-1477.

Chen, T. and Sun, Y. (2017). Task-guided and path-augmented heterogeneous network embedding for author identification. In Proceedings of the Tenth ACM International Conference on Web Search and Data Mining, pages 295-304. ACM.

Chen, Y., Rohrbach, M., Yan, Z., Shuicheng, Y., Feng, J., and Kalantidis, Y. (2019c). Graph-based global reasoning networks. In The IEEE Conference on Computer Vision and Pattern Recognition (CVPR). IEEE.

Chen, Y., Sun, K., Pu, J., Xiong, Z., and Zhang, X. (2020). Grapasa: Parametric graph embedding via siamese architecture. Information Sciences, 512:1442-1457.

Cheng, W., Zhang, X., Guo, Z., Wu, Y., Sullivan, P. F., and Wang, W. (2013). Flexible and robust co-regularized multi-domain graph clustering. In Proceedings of the 19th ACM SIGKDD international conference on Knowledge discovery and data mining, pages 320-328. ACM, ACM.

Chiang, W.-L., Liu, X., Si, S., Li, Y., Bengio, S., and Hsieh, C.-J. (2019). Cluster-gen: An efficient algorithm for training deep and large graph convolutional networks. In Proceedings of the 25th ACM SIGKDD International Conference on Knowledge Discovery \& Data Mining, pages 257-266.

Cho, H. and Yu, Y. (2018). Link prediction for interdisciplinary collaboration via co-authorship network. Social Network Analysis and Mining, 8(1):25.

Chung, F. R. and Graham, F. C. (1997). Spectral graph theory, volume 92. American Mathematical Soc.

Clauset, A., Moore, C., and Newman, M. E. (2008). Hierarchical structure and the prediction of missing links in networks. Nature, 453(7191):98.

Cobanoglu, M. C., Liu, C., Hu, F., Oltvai, Z. N., and Bahar, I. (2013). Predicting drug-target interactions using probabilistic matrix factorization. Journal of chemical information and modeling, 53(12):33993409.

Crichton, G., Guo, Y., Pyysalo, S., and Korhonen, A. (2018). Neural networks for link prediction in realistic biomedical graphs: a multi-dimensional evaluation of graph embedding-based approaches. BMC bioinformatics, 19(1):176.

Cui, P., Wang, X., Pei, J., and Zhu, W. (2018). A survey on network embedding. IEEE Transactions on Knowledge and Data Engineering, 31(5):833-852.

Dai, H., Dai, B., and Song, L. (2016). Discriminative embeddings of latent variable models for structured data. In International conference on machine learning, pages 2702-2711. ACM.

Dai, H., Li, H., Tian, T., Huang, X., Wang, L., Zhu, J., and Song, L. (2018). Adversarial attack on graph structured data. arXiv preprint arXiv:1806.02371, 1806.02371.

De Oliveira, M. F. and Levkowitz, H. (2003). From visual data exploration to visual data mining: a survey. IEEE Transactions on Visualization and Computer Graphics, 9(3):378-394.

Deerwester, S., Dumais, S. T., Furnas, G. W., Landauer, T. K., and Harshman, R. (1990). Indexing by latent semantic analysis. Journal of the American society for information science, 41(6):391-407.

Defferrard, M., Bresson, X., and Vandergheynst, P. (2016). Convolutional neural networks on graphs with fast localized spectral filtering. In Advances in neural information processing systems, pages 3844-3852.

Devlin, J., Chang, M.-W., Lee, K., and Toutanova, K. (2018). Bert: Pre-training of deep bidirectional transformers for language understanding. arXiv preprint arXiv:1810.04805, 1810.04805.

Didimo, W., Liotta, G., and Montecchiani, F. (2019). A survey on graph drawing beyond planarity. ACM Comput. Surv., 52(1):4:1-4:37.

Ding, C. H., He, X., Zha, H., Gu, M., and Simon, H. D. (2001). A min-max cut algorithm for graph partitioning and data clustering. In Proceedings 2001 IEEE International Conference on Data Mining, pages 107-114. IEEE. 
Ding, M., Tang, J., and Zhang, J. (2018). Semi-supervised learning on graphs with generative adversarial nets. In Proceedings of the 27th ACM International Conference on Information and Knowledge Management, pages 913-922. ACM.

Do, D. T., Le, T. Q. T., and Le, N. Q. K. (2020). Using deep neural networks and biological subwords to detect protein s-sulfenylation sites. Briefings in Bioinformatics.

Dong, Y., Chawla, N. V., and Swami, A. (2017). metapath2vec: Scalable representation learning for heterogeneous networks. In Proceedings of the 23rd ACM SIGKDD international conference on knowledge discovery and data mining, pages 135-144. ACM.

Donnat, C., Holmes, S., et al. (2018a). Tracking network dynamics: A survey using graph distances. The Annals of Applied Statistics, 12(2):971-1012.

Donnat, C., Zitnik, M., Hallac, D., and Leskovec, J. (2018b). Learning structural node embeddings via diffusion wavelets. In Proceedings of the 24th ACM SIGKDD International Conference on Knowledge Discovery \& Data Mining, volume 24, pages 1320-1329. ACM.

Duvenaud, D. K., Maclaurin, D., Iparraguirre, J., Bombarell, R., Hirzel, T., Aspuru-Guzik, A., and Adams, R. P. (2015). Convolutional networks on graphs for learning molecular fingerprints. In Advances in neural information processing systems, pages 2224-2232. MIT press.

Efron, B. (1992). Bootstrap methods: another look at the jackknife. In Breakthroughs in statistics, pages 569-593. Springer International Publishing.

Erdös, P. and Rényi, A. (1959). On random graphs publ. Math. debrecen, 6:290-297.

Ezzat, A., Wu, M., Li, X.-L., and Kwoh, C.-K. (2017). Drug-target interaction prediction using ensemble learning and dimensionality reduction. Methods, 129:81-88.

Ezzat, A., Zhao, P., Wu, M., Li, X.-L., and Kwoh, C.-K. (2016). Drug-target interaction prediction with graph regularized matrix factorization. IEEE/ACM transactions on computational biology and bioinformatics, 14(3):646-656.

Fan, M., Zhou, Q., Chang, E., and Zheng, T. F. (2014). Transition-based knowledge graph embedding with relational mapping properties. In Proceedings of the 28th Pacific Asia Conference on Language, Information and Computing, volume 28, pages 328-337. Association for Computational Linguistics.

Fang, H., Wu, F., Zhao, Z., Duan, X., Zhuang, Y., and Ester, M. (2016). Community-based question answering via heterogeneous social network learning. In Thirtieth AAAI Conference on Artificial Intelligence, pages 122-128.

Fathy, A. and Li, K. (2020). Temporalgat: Attention-based dynamic graph representation learning. In Lauw, H. W., Wong, R. C.-W., Ntoulas, A., Lim, E.-P., Ng, S.-K., and Pan, S. J., editors, Advances in Knowledge Discovery and Data Mining, pages 413-423, Cham. Springer International Publishing.

Feng, R., Yang, Y., Hu, W., Wu, F., and Zhang, Y. (2018). Representation learning for scale-free networks. In Thirty-Second AAAI Conference on Artificial Intelligence.

Fey, M., Eric Lenssen, J., Weichert, F., and Müller, H. (2018). Splinecnn: Fast geometric deep learning with continuous b-spline kernels. In Proceedings of the IEEE Conference on Computer Vision and Pattern Recognition, pages 869-877.

Fortunato, S. (2010). Community detection in graphs. Physics reports, 486(3-5):75-174.

Fu, X., Zhang, J., Meng, Z., and King, I. (2020). Magnn: Metapath aggregated graph neural network for heterogeneous graph embedding. In Proceedings of The Web Conference 2020, WWW'20, page 2331-2341, New York, NY, USA. Association for Computing Machinery.

Gallicchio, C. and Micheli, A. (2020). Fast and deep graph neural networks. In AAAI, pages 3898-3905. AAAI.

Ganguly, S., Gupta, M., Varma, V., Pudi, V., et al. (2016). Author2vec: Learning author representations by combining content and link information. In Proceedings of the 25th International Conference Companion on World Wide Web, pages 49-50. International World Wide Web Conferences Steering Committee.

Gao, F., Musial, K., Cooper, C., and Tsoka, S. (2015). Link prediction methods and their accuracy for different social networks and network metrics. Scientific Programming, 2015:1.

Gao, J., Zhang, T., and Xu, C. (2019). Graph convolutional tracking. In CVPR.

Gao, S., Denoyer, L., and Gallinari, P. (2011). Temporal link prediction by integrating content and structure information. In Proceedings of the 20th ACM International Conference on Information and Knowledge Management, CIKM '11, pages 1169-1174, New York, NY, USA. ACM.

Geng, X., Zhang, H., Bian, J., and Chua, T.-S. (2015). Learning image and user features for recommen- 
dation in social networks. In Proceedings of the IEEE International Conference on Computer Vision, pages 4274-4282.

Getoor, L. and Taskar, B. (2007). Statistical relational learning.

Gilmer, J., Schoenholz, S. S., Riley, P. F., Vinyals, O., and Dahl, G. E. (2017). Neural message passing for quantum chemistry. In Proceedings of the 34th International Conference on Machine Learning-Volume 70, pages 1263-1272. JMLR. org.

Golub, G. H. and Reinsch, C. (1971). Singular value decomposition and least squares solutions. In Linear Algebra, pages 134-151. Springer.

Goyal, P., Chhetri, S. R., and Canedo, A. (2020). dyngraph2vec: Capturing network dynamics using dynamic graph representation learning. Knowledge-Based Systems, 187:104816.

Goyal, P. and Ferrara, E. (2017). Graph embedding techniques, applications, and performance: A survey. arXiv preprint arXiv:1705.02801, 1705.02801.

Goyal, P., Hosseinmardi, H., Ferrara, E., and Galstyan, A. (2018). Embedding networks with edge attributes. In Proceedings of the 29th on Hypertext and Social Media, pages 38-42. ACM.

Grover, A. and Leskovec, J. (2016). node2vec: Scalable feature learning for networks. In Proceedings of the 22nd ACM SIGKDD IC on KDD, volume 22, pages 855-864. ACM, ACM.

Gui, H., Liu, J., Tao, F., Jiang, M., Norick, B., and Han, J. (2016). Large-scale embedding learning in heterogeneous event data. In 2016 IEEE 16th International Conference on Data Mining (ICDM), pages 907-912. IEEE.

Guo, Z., Zhang, Y., and Lu, W. (2019). Attention guided graph convolutional networks for relation extraction. In Proceedings of the 57th Annual Meeting of the Association for Computational Linguistics, pages 241-251, Florence, Italy. Association for Computational Linguistics.

Haddad, M., Bothorel, C., Lenca, P., and Bedart, D. (2019). Temporalnode2vec: Temporal node embedding in temporal networks. In International Conference on Complex Networks and Their Applications, pages 891-902. Springer International Publishing.

Hamilton, W., Ying, Z., and Leskovec, J. (2017a). Inductive representation learning on large graphs. In Advances in NIPS, pages 1025-1035. MIT press.

Hamilton, W. L., Ying, R., and Leskovec, J. (2017b). Representation learning on graphs: Methods and applications. arXiv preprint arXiv:1709.05584, 1709.05584.

Hasan, M. A. and Zaki, M. J. (2011). A Survey of Link Prediction in Social Networks, chapter 9, pages 243-275. Springer US, Boston, MA.

Hayashi, K. and Ohsaki, M. (2020). Reinforcement learning and graph embedding for binary truss topology optimization under stress and displacement constraints. Frontiers in Built Environment, 6:59.

He, Q., Pei, J., Kifer, D., Mitra, P., and Giles, L. (2010). Context-aware citation recommendation. In Proceedings of the 19th International Conference on World Wide Web, WWW '10, pages 421-430, New York, NY, USA. ACM.

He, S., Liu, K., Ji, G., and Zhao, J. (2015). Learning to represent knowledge graphs with gaussian embedding. In Proceedings of the 24th ACM International on Conference on Information and Knowledge Management, volume 24, pages 623-632. ACM, ACM.

$\mathrm{He}, \mathrm{X}$. and Niyogi, P. (2004). Locality preserving projections. In Advances in neural information processing systems, pages 153-160.

Heckerman, D., Meek, C., and Koller, D. (2007). Probabilistic entity-relationship models, prms, and plate models. Introduction to statistical relational learning, pages 201-238.

Henaff, M., Bruna, J., and LeCun, Y. (2015). Deep convolutional networks on graph-structured data. arXiv preprint arXiv:1506.05163, 1506.05163.

Herman, I., Melançon, G., and Marshall, M. S. (2000). Graph visualization and navigation in information visualization: A survey. IEEE Transactions on visualization and computer graphics, 6(1):24-43.

Hettige, B., Wang, W., Li, Y.-F., and Buntine, W. (2020). Robust attribute and structure preserving graph embedding. In Pacific-Asia Conference on Knowledge Discovery and Data Mining, pages 593-606. Springer, Springer International Publishing.

Hong, H., Lin, Y., Yang, X., Li, Z., Fu, K., Wang, Z., Qie, X., and Ye, J. (2020). Heteta: Heterogeneous information network embedding for estimating time of arrival. In Proceedings of the 26th ACM SIGKDD International Conference on Knowledge Discovery \& Data Mining, KDD '20, page 2444-2454, New York, NY, USA. Association for Computing Machinery.

Hou, C., Nie, F., Tao, H., and Yi, D. (2017). Multi-view unsupervised feature selection with adaptive 
similarity and view weight. IEEE Transactions on Knowledge and Data Engineering, 29(9):1998-2011. Hu, B., Fang, Y., and Shi, C. (2019). Adversarial learning on heterogeneous information networks. In Proceedings of the 25th ACM SIGKDD International Conference on Knowledge Discovery \& Data Mining, KDD '19, page 120-129, New York, NY, USA. Association for Computing Machinery.

Huang, X., Li, J., and Hu, X. (2017). Label informed attributed network embedding. In Proceedings of the Tenth ACM International Conference on Web Search and Data Mining, pages 731-739. ACM.

Huang, X., Zhang, J., Li, D., and Li, P. (2019). Knowledge graph embedding based question answering. In Proceedings of the Twelfth ACM International Conference on Web Search and Data Mining, volume 12 of WSDM '19, pages 105-113, New York, NY, USA. ACM.

Huang, Z. and Mamoulis, N. (2017). Heterogeneous information network embedding for meta path based proximity. arXiv preprint arXiv:1701.05291, 1701.05291.

Islam, M. R., Prakash, B. A., and Ramakrishnan, N. (2018). Signet: Scalable embeddings for signed networks. In Pacific-Asia Conference on Knowledge Discovery and Data Mining, pages 157-169. Springer International Publishing.

Jacob, Y., Denoyer, L., and Gallinari, P. (2014). Learning latent representations of nodes for classifying in heterogeneous social networks. In Proceedings of the 7th ACM international conference on Web search and data mining, pages 373-382. ACM.

Jenatton, R., Roux, N. L., Bordes, A., and Obozinski, G. R. (2012). A latent factor model for highly multi-relational data. In Advances in Neural Information Processing Systems, pages 3167-3175. MIT press.

Ji, G., He, S., Xu, L., Liu, K., and Zhao, J. (2015). Knowledge graph embedding via dynamic mapping matrix. In Proceedings of the 53rd Annual Meeting of the Association for Computational Linguistics and the 7th International Joint Conference on Natural Language Processing (Volume 1: Long Papers), volume 53, pages 687-696. Association for Computational Linguistics.

Ji, G., Liu, K., He, S., and Zhao, J. (2016). Knowledge graph completion with adaptive sparse transfer matrix. In Thirtieth AAAI Conference on Artificial Intelligence, volume 30. AAAI.

Jiang, Z., Gao, Z., Lan, J., Yang, H., Lu, Y., and Liu, X. (2020). Task-oriented genetic activation for large-scale complex heterogeneous graph embedding. In Proceedings of The Web Conference 2020, WWW '20, page 1581-1591, New York, NY, USA. Association for Computing Machinery.

Jing, Y., Wang, H., Shao, K., Huo, X., and Zhang, Y. (2020). Unsupervised graph representation learning with variable heat kernel. IEEE Access, 8:15800-15811.

Johansson, F. D. and Dubhashi, D. (2015). Learning with similarity functions on graphs using matchings of geometric embeddings. In Proceedings of the 21th ACM SIGKDD International Conference on Knowledge Discovery and Data Mining, pages 467-476. ACM.

Kearnes, S., McCloskey, K., Berndl, M., Pande, V., and Riley, P. (2016). Molecular graph convolutions: moving beyond fingerprints. Journal of computer-aided molecular design, 30(8):595-608.

Kefato, Z. T., Sheikh, N., and Montresor, A. (2020). Which way? direction-aware attributed graph embedding.

Keser, R., Nallbani, I., Calik, N., Ayanzadeh, A., and Töreyin, B. (2020). Graph embedding for link prediction using residual variational graph autoencoders. In THE 28th IEEE CONFERENCE ON SIGNAL PROCESSING AND COMMUNICATIONS APPLICATIONS. IEEE.

Khalil, E., Dai, H., Zhang, Y., Dilkina, B., and Song, L. (2017). Learning combinatorial optimization algorithms over graphs. In Advances in Neural Information Processing Systems, pages 6348-6358.

Khasahmadi, A. H., Hassani, K., Moradi, P., Lee, L., and Morris, Q. (2020). Memory-based graph networks. In International Conference on Learning Representations.

Kim, D., Kim, S., and Kwak, N. (2018a). Textbook question answering with knowledge graph understanding and unsupervised open-set text comprehension. CoRR, abs/1811.00232.

Kim, J., Kim, T., Kim, S., and Yoo, C. D. (2019). Edge-labeling graph neural network for few-shot learning. CoRR, abs/1905.01436.

Kim, J., Park, H., Lee, J.-E., and Kang, U. (2018b). Side: Representation learning in signed directed networks. In Proceedings of the 2018 World Wide Web Conference, WWW'18, page 509-518, Republic and Canton of Geneva, CHE. International World Wide Web Conferences Steering Committee.

Kingma, D. P. and Welling, M. (2013). Auto-encoding variational bayes. arXiv preprint arXiv:1312.6114, 1312.6114.

Kipf, T. N. and Welling, M. (2016a). Semi-supervised classification with graph convolutional networks. 
arXiv preprint arXiv:1609.02907, 1609.02907.

Kipf, T. N. and Welling, M. (2016b). Variational graph auto-encoders. arXiv preprint arXiv:1611.07308, 1611.07308 .

Kleinberg, R. (2007). Geographic routing using hyperbolic space. In IEEE INFOCOM 2007-26th IEEE International Conference on Computer Communications, pages 1902-1909. IEEE.

Kolouri, S., Naderializadeh, N., Rohde, G. K., and Hoffmann, H. (2020). Wasserstein embedding for graph learning.

Kong, X., Mao, M., Wang, W., Liu, J., and Xu, B. (2018). Voprec: Vector representation learning of papers with text information and structural identity for recommendation. IEEE Transactions on Emerging Topics in Computing.

Krioukov, D., Papadopoulos, F., Boguñá, M., and Vahdat, A. (2009). Greedy forwarding in scale-free networks embedded in hyperbolic metric spaces. ACM SIGMETRICS Performance Evaluation Review, 37(2):15-17.

Kruskal, J. and Wish, M. (1978). Multidimensional Scaling. Number no. 11 in 07. SAGE Publications.

Kulmanov, M., Khan, M. A., and Hoehndorf, R. (2018). Deepgo: predicting protein functions from sequence and interactions using a deep ontology-aware classifier. Bioinformatics, 34(4):660-668.

Laakom, F., Raitoharju, J., Passalis, N., Iosifidis, A., and Gabbouj, M. (2020). Graph embedding with data uncertainty. arXiv preprint arXiv:2009.00505, 2009.00505.

Le, N. Q. K., Yapp, E. K. Y., and Yeh, H.-Y. (2019). Et-gru: using multi-layer gated recurrent units to identify electron transport proteins. BMC bioinformatics, 20(1):377.

Le, T. M. and Lauw, H. W. (2014). Probabilistic latent document network embedding. In 2014 IEEE International Conference on Data Mining (ICDM), pages 270-279. IEEE.

Lee, J. B., Rossi, R., and Kong, X. (2018). Graph classification using structural attention. In Proceedings of the 24th ACM SIGKDD International Conference on Knowledge Discovery \& Data Mining, pages 1666-1674. ACM.

Lee, J. B., Rossi, R. A., Kim, S., Ahmed, N. K., and Koh, E. (2019). Attention models in graphs: A survey. ACM Trans. Knowl. Discov. Data, 13(6).

Lei, M., Shi, Y., and Niu, L. (2018). The applications of stochastic models in network embedding: A survey. In 2018 IEEE/WIC/ACM International Conference on Web Intelligence (WI), pages 635-638. IEEE.

Leskovec, J., Huttenlocher, D., and Kleinberg, J. (2010). Signed networks in social media. In Proceedings of the SIGCHI Conference on Human Factors in Computing Systems, CHI '10, page 1361-1370, New York, NY, USA. Association for Computing Machinery.

Levie, R., Monti, F., Bresson, X., and Bronstein, M. M. (2017). Cayleynets: Graph convolutional neural networks with complex rational spectral filters. IEEE Transactions on Signal Processing, 67(1):97-109.

Li, B., Pi, D., Lin, Y., Khan, I. A., and Cui, L. (2020). Multi-source information fusion based heterogeneous network embedding. Information Sciences.

Li, G., Luo, J., Xiao, Q., Liang, C., Ding, P., and Cao, B. (2017a). Predicting microrna-disease associations using network topological similarity based on deepwalk. Ieee Access, 5:24032-24039.

Li, J., Chen, C., Tong, H., and Liu, H. (2018). Multi-layered network embedding. In Proceedings of the 2018 SIAM International Conference on Data Mining, pages 684-692. SIAM, SIAM.

Li, J., Ritter, A., and Jurafsky, D. (2015a). Learning multi-faceted representations of individuals from heterogeneous evidence using neural networks. arXiv preprint arXiv:1510.05198, 1510.05198.

Li, J., Zhu, J., and Zhang, B. (2016). Discriminative deep random walk for network classification. In Proceedings of the 54th Annual Meeting of the Association for Computational Linguistics (Volume 1: Long Papers), volume 1, pages 1004-1013. Association for Computational Linguistics.

Li, K., Gao, J., Guo, S., Du, N., Li, X., and Zhang, A. (2014a). Lrbm: A restricted boltzmann machine based approach for representation learning on linked data. In 2014 IEEE International Conference on Data Mining, pages 300-309. IEEE, IEEE.

Li, M., Chen, S., Chen, X., Zhang, Y., Wang, Y., and Tian, Q. (2019a). Actional-structural graph convolutional networks for skeleton-based action recognition. In The IEEE Conference on Computer Vision and Pattern Recognition (CVPR). IEEE.

Li, Q., Shang, Y., Qiao, X., and Dai, W. (2020). Heterogeneous dynamic graph attention network. In 2020 IEEE International Conference on Knowledge Graph (ICKG), pages 404-411. IEEE.

Li, W., Xu, J., He, Y., Yan, S., Wu, Y., and Sun, X. (2019b). Coherent comments generation for Chinese 
articles with a graph-to-sequence model. In Proceedings of the 57th Annual Meeting of the Association for Computational Linguistics, pages 4843-4852, Florence, Italy. Association for Computational Linguistics.

Li, X. and Chen, H. (2009). Recommendation as link prediction: A graph kernel-based machine learning approach. In Proceedings of the 9th ACM/IEEE-CS Joint Conference on Digital Libraries, JCDL '09, pages 213-216, New York, NY, USA. ACM.

Li, X., Chen, W., Chen, Y., Zhang, X., Gu, J., and Zhang, M. Q. (2017b). Network embedding-based representation learning for single cell rna-seq data. Nucleic acids research, 45(19):e166-e166.

Li, X., Du, N., Li, H., Li, K., Gao, J., and Zhang, A. (2014b). A deep learning approach to link prediction in dynamic networks. In Proceedings of the 2014 SIAM International Conference on Data Mining, pages 289-297. SIAM, SIAM.

Li, Y., Nie, F., Huang, H., and Huang, J. (2015b). Large-scale multi-view spectral clustering via bipartite graph. In Proceedings of the twenty-ninth AAAI conference on artificial intelligence, volume 29, pages 2750-2756. AAAI.

Li, Y., Tarlow, D., Brockschmidt, M., and Zemel, R. (2015c). Gated graph sequence neural networks. arXiv preprint arXiv:1511.05493, 1511.05493.

Li, Y., Yu, R., Shahabi, C., and Liu, Y. (2017c). Diffusion convolutional recurrent neural network: Data-driven traffic forecasting. arXiv preprint arXiv:1707.01926, 1707.01926.

Liben-Nowell, D. and Kleinberg, J. (2007). The link-prediction problem for social networks. journal of the Association for Information Science and Technology, 58(7):1019-1031.

Lim, K. W. and Buntine, W. L. (2016). Bibliographic analysis with the citation network topic model. CoRR, abs/1609.06826.

Lin, B., Ghaddar, B., and Nathwani, J. (2020a). Deep reinforcement learning for electric vehicle routing problem with time windows. arXiv preprint arXiv:2010.02068, 2010.02068.

Lin, Y., Hou, J., Laurienti, P. J., and Wu, G. (2020b). Detecting changes of functional connectivity by dynamic graph embedding learning. In Martel, A. L., Abolmaesumi, P., Stoyanov, D., Mateus, D., Zuluaga, M. A., Zhou, S. K., Racoceanu, D., and Joskowicz, L., editors, Medical Image Computing and Computer Assisted Intervention - MICCAI 2020, pages 489-497, Cham. Springer International Publishing.

Lin, Y., Liu, Z., Sun, M., Liu, Y., and Zhu, X. (2015). Learning entity and relation embeddings for knowledge graph completion. In Twenty-ninth AAAI conference on artificial intelligence, volume 39, pages 2181-2187. AAAI.

Lin, Y.-Y., Liu, T.-L., and Chen, H.-T. (2005). Semantic manifold learning for image retrieval. In Proceedings of the 13th annual ACM international conference on Multimedia, pages 249-258. ACM.

Liu, A. (2020). Anonymized gcn: A novel robust graph embedding method via hiding node position in noise. arXiv preprint arXiv:2005.03482, 2005.03482.

Liu, F., Liu, B., Sun, C., Liu, M., and Wang, X. (2013). Deep learning approaches for link prediction in social network services. In International Conference on Neural Information Processing, pages 425-432. Springer International Publishing.

Liu, F., Liu, B., Sun, C., Liu, M., and Wang, X. (2015a). Deep belief network-based approaches for link prediction in signed social networks. Entropy, 17(4):2140-2169.

Liu, J., Xu, C., Yin, C., Wu, W., and Song, Y. (2020a). K-core based temporal graph convolutional network for dynamic graphs. arXiv preprint arXiv:2003.09902, 2003.09902.

Liu, L., Cheung, W. K., Li, X., and Liao, L. (2016). Aligning users across social networks using network embedding. In IJCAI, pages 1774-1780.

Liu, R., Cheng, W., Tong, H., Wang, W., and Zhang, X. (2015b). Robust multi-network clustering via joint cross-domain cluster alignment. In 2015 IEEE International Conference on Data Mining, pages 291-300. IEEE, IEEE.

Liu, W., Chen, P.-Y., Yeung, S., Suzumura, T., and Chen, L. (2017a). Principled multilayer network embedding. In 2017 IEEE International Conference on Data Mining Workshops (ICDMW), pages 134-141. IEEE, IEEE.

Liu, X., Li, Q., Shen, C., Peng, X., Zhou, Y., and Guan, X. (2020b). Learning by sampling and compressing: Efficient graph representation learning with extremely limited annotations.

Liu, X., Murata, T., Kim, K.-S., Kotarasu, C., and Zhuang, C. (2019). A general view for network embedding as matrix factorization. In Proceedings of the Twelfth ACM International Conference on 
Web Search and Data Mining, volume 12 of WSDM '19, pages 375-383, New York, NY, USA. ACM. Liu, Y. and Kou, Z. (2007). Predicting who rated what in large-scale datasets. SIGKDD Explor. Newsl., 9(2):62-65.

Liu, Y., Zhang, X., Liu, L., and Li, G. (2020c). Graph embedding based on characteristic of rooted subgraph structure. In Li, G., Shen, H. T., Yuan, Y., Wang, X., Liu, H., and Zhao, X., editors, Knowledge Science, Engineering and Management, pages 28-39, Cham. Springer International Publishing.

Liu, Z., Chen, C., Li, L., Zhou, J., Li, X., Song, L., and Qi, Y. (2018a). Geniepath: Graph neural networks with adaptive receptive paths. arXiv preprint arXiv:1802.00910, 1802.00910.

Liu, Z., Zheng, V. W., Zhao, Z., Zhu, F., Chang, K. C.-C., Wu, M., and Ying, J. (2017b). Semantic proximity search on heterogeneous graph by proximity embedding. In AAAI, pages 154-160.

Liu, Z., Zheng, V. W., Zhao, Z., Zhu, F., Chang, K. C.-C., Wu, M., and Ying, J. (2018b). Distance-aware dag embedding for proximity search on heterogeneous graphs. In Thirty-Second AAAI Conference on Artificial Intelligence, pages 2355-2362. AAAI.

Lu, C., Jiao, P., Liu, H., Wang, Y., Xu, H., and Wang, W. (2019). Ssne: Status signed network embedding. In Yang, Q., Zhou, Z.-H., Gong, Z., Zhang, M.-L., and Huang, S.-J., editors, Advances in Knowledge Discovery and Data Mining, pages 81-93, Cham. Springer International Publishing.

Lü, L. and Zhou, T. (2011). Link prediction in complex networks: A survey. Physica A: statistical mechanics and its applications, 390(6):1150-1170.

Lu, P.-E. and Chang, C.-S. (2020). Explainable, stable, and scalable graph convolutional networks for learning graph representation. arXiv preprint arXiv:2009.10367, 2009.10367.

Lu, Q. and Getoor, L. (2003). Link-based classification. In Proceedings of the 20th International Conference on Machine Learning (ICML-03), pages 496-503. ACM.

Luo, D., Nie, F., Huang, H., and Ding, C. H. (2011). Cauchy graph embedding. In Proceedings of the 28th International Conference on Machine Learning (ICML-11), pages 553-560.

Luo, H., Li, Y., Fu, J., and Glass, J. (2019). Language modeling with graph temporal convolutional networks.

Luo, Y., Zhao, X., Zhou, J., Yang, J., Zhang, Y., Kuang, W., Peng, J., Chen, L., and Zeng, J. (2017). A network integration approach for drug-target interaction prediction and computational drug repositioning from heterogeneous information. Nature communications, 8(1):1-13.

Lv, X., Hou, L., Li, J., and Liu, Z. (2018). Differentiating concepts and instances for knowledge graph embedding. arXiv preprint arXiv:1811.04588, 1811.04588.

Maaten, L. v. d. and Hinton, G. (2008). Visualizing data using t-sne. Journal of machine learning research, 9(Nov):2579-2605.

Mai, G., Janowicz, K., and Yan, B. (2018). Support and centrality: Learning weights for knowledge graph embedding models. In European Knowledge Acquisition Workshop, pages 212-227. Springer International Publishing.

Makarov, I., Gerasimova, O., Sulimov, P., Korovina, K., and Zhukov, L. (2019a). Joint node-edge network embedding for link prediction. In Springer Proceedings in Mathematics and Statistic (to appear), pages 1-12. Springer International Publishing.

Makarov, I., Gerasimova, O., Sulimov, P., and Zhukov, L. (2019b). Co-authorship network embedding and recommending collaborators via network embedding. In Springer Proceedings in Mathematics and Statistic (to appear), pages 1-6. Springer International Publishing.

Makarov, I., Gerasimova, O., Sulimov, P., and Zhukov, L. E. (2018). Recommending co-authorship via network embeddings and feature engineering. In Proceedings of the 18th ACM/IEEE JCDL, pages 1-2. ACM.

Makarov, I., Gerasimova, O., Sulimov, P., and Zhukov, L. E. (2019c). Dual network embedding for representing research interests in the link prediction problem on co-authorship networks. PeerJ Computer Science, 5:e172.

Malliaros, F. D. and Vazirgiannis, M. (2013). Clustering and community detection in directed networks: A survey. Physics Reports, 533(4):95-142.

Marcheggiani, D., Bastings, J., and Titov, I. (2018). Exploiting semantics in neural machine translation with graph convolutional networks. arXiv preprint arXiv:1804.08313, 1804.08313.

Marcheggiani, D. and Titov, I. (2017). Encoding sentences with graph convolutional networks for semantic role labeling. arXiv preprint arXiv:1703.04826, 1703.04826.

Martínez, A. M. and Kak, A. C. (2001). Pca versus lda. IEEE transactions on pattern analysis and 
machine intelligence, 23(2):228-233.

McInnes, L., Healy, J., and Melville, J. (2018). Umap: Uniform manifold approximation and projection for dimension reduction. arXiv preprint arXiv:1802.03426, 1802.03426.

McPherson, M., Smith-Lovin, L., and Cook, J. M. (2001). Birds of a feather: Homophily in social networks. Annual review of sociology, 27(1):415-444.

Mikolov, T., Sutskever, I., Chen, K., Corrado, G. S., and Dean, J. (2013). Distributed representations of words and phrases and their compositionality. In Advances in NIPS, pages 3111-3119. MIT press.

Monti, F., Boscaini, D., Masci, J., Rodola, E., Svoboda, J., and Bronstein, M. M. (2017a). Geometric deep learning on graphs and manifolds using mixture model cnns. In Proceedings of the IEEE Conference on Computer Vision and Pattern Recognition, pages 5115-5124.

Monti, F., Bronstein, M., and Bresson, X. (2017b). Geometric matrix completion with recurrent multigraph neural networks. In Advances in Neural Information Processing Systems, pages 3697-3707.

Monti, F., Shchur, O., Bojchevski, A., Litany, O., Günnemann, S., and Bronstein, M. M. (2018). Dualprimal graph convolutional networks. arXiv preprint arXiv:1806.00770, 1806.00770.

Mousavi, S. F., Safayani, M., Mirzaei, A., and Bahonar, H. (2017). Hierarchical graph embedding in vector space by graph pyramid. Pattern Recognition, 61:245-254.

Moyano, L. G. (2017). Learning network representations. The European Physical Journal Special Topics, 226(3):499-518.

Narayanan, A., Chandramohan, M., Chen, L., Liu, Y., and Saminathan, S. (2016). subgraph2vec: Learning distributed representations of rooted sub-graphs from large graphs. arXiv preprint arXiv:1606.08928, 1606.08928 .

Natarajan, N. and Dhillon, I. S. (2014). Inductive matrix completion for predicting gene-disease associations. Bioinformatics, 30(12):i60-i68.

Navlakha, S., Rastogi, R., and Shrivastava, N. (2008). Graph summarization with bounded error. In Proceedings of the 2008 ACM SIGMOD international conference on Management of data, pages 419-432. ACM.

Newman, M. E. (2005). A measure of betweenness centrality based on random walks. Social networks, 27(1):39-54.

Newman, M. E. and Girvan, M. (2004). Finding and evaluating community structure in networks. Physical review E, 69(2):026113.

Ni, J., Cheng, W., Fan, W., and Zhang, X. (2016). Self-grouping multi-network clustering. In 2016 IEEE 16th International Conference on Data Mining (ICDM), pages 1119-1124. IEEE, IEEE.

Nickel, M., Murphy, K., Tresp, V., and Gabrilovich, E. (2016). A review of relational machine learning for knowledge graphs. Proceedings of the IEEE, 104(1):11-33.

Nickel, M., Rosasco, L., and Poggio, T. (2015). Holographic embeddings of knowledge graphs. arXiv preprint arXiv:1510.04935, 1510.04935 .

Nickel, M., Tresp, V., and Kriegel, H.-P. (2011). A three-way model for collective learning on multirelational data. In $I C M L$, volume 11, pages 809-816. ACM.

Nie, F., Cai, G., and Li, X. (2017a). Multi-view clustering and semi-supervised classification with adaptive neighbours. In Thirty-First AAAI Conference on Artificial Intelligence, volume 31. AAAI.

Nie, F., Zhu, W., and Li, X. (2017b). Unsupervised large graph embedding. In AAAI, pages 2422-2428.

Nie, F., Zhu, W., and Li, X. (2020). Unsupervised large graph embedding based on balanced and hierarchical k-means. IEEE Transactions on Knowledge and Data Engineering, pages 1-1.

Niepert, M., Ahmed, M., and Kutzkov, K. (2016). Learning convolutional neural networks for graphs. In International conference on machine learning, pages 2014-2023.

Nikolentzos, G., Meladianos, P., and Vazirgiannis, M. (2017). Matching node embeddings for graph similarity. In $A A A I$, pages 2429-2435.

Nozza, D., Fersini, E., and Messina, E. (2020). Cage: Constrained deep attributed graph embedding. Information Sciences, 518:56-70.

Ortega, A., Frossard, P., Kovačević, J., Moura, J. M., and Vandergheynst, P. (2018a). Graph signal processing: Overview, challenges, and applications. Proceedings of the IEEE, 106(5):808-828.

Ortega, F., Bobadilla, J., Gutiérrez, A., Hurtado, R., and Li, X. (2018b). Artificial intelligence scientific documentation dataset for recommender systems. IEEE Access, 6:48543-48555.

Ou, M., Cui, P., Pei, J., Zhang, Z., and Zhu, W. (2016). Asymmetric transitivity preserving graph embedding. In Proc. of the 22nd ACM SIGKDD IC on KDD, volume 22, pages 1105-1114. ACM, 
ACM.

Pan, S., Hu, R., Fung, S.-f., Long, G., Jiang, J., and Zhang, C. (2019). Learning graph embedding with adversarial training methods. arXiv preprint arXiv:1901.01250, 1901.01250.

Pan, S., Wu, J., Zhu, X., Zhang, C., and Wang, Y. (2016). Tri-party deep network representation. Network, 11(9): 12.

Pardalos, P. M. and Xue, J. (1994). The maximum clique problem. Journal of global Optimization, 4(3):301-328.

Pennington, J., Socher, R., and Manning, C. (2014). Glove: Global vectors for word representation. In Proceedings of the 2014 conference on empirical methods in natural language processing (EMNLP), pages $1532-1543$.

Perozzi, B., Al-Rfou, R., and Skiena, S. (2014). Deepwalk: Online learning of social representations. In Proceedings of the 20th ACM SIGKDD IC on KDD, volume 20, pages 701-710. ACM, ACM.

Perozzi, B., Kulkarni, V., Chen, H., and Skiena, S. (2017). Don't walk, skip!: Online learning of multi-scale network embeddings. In Proceedings of the 2017 IEEE/ACM International Conference on Advances in Social Networks Analysis and Mining 2017, pages 258-265. ACM.

Perozzi, B., Kulkarni, V., and Skiena, S. (2016). Walklets: Multiscale graph embeddings for interpretable network classification. CoRR, abs/1605.02115.

Phuc, L. H., Yamada, M., and Kashima, H. (2020). Link prediction on multiple graphs with graph embedding and optimal transport. In Proceedings of the National Conference of the Society for Artificial Intelligence, 34th National Conference (2020), pages 4Rin189-4Rin189. JSAI.

Pimentel, T., Veloso, A., and Ziviani, N. (2017). Unsupervised and scalable algorithm for learning node representations. Proceedings of ICLR Workshop Track, pages 1-4.

Pirotte, A., Renders, J.-M., Saerens, M., et al. (2007). Random-walk computation of similarities between nodes of a graph with application to collaborative recommendation. IEEE Transactions on Knowledge \& Data Engineering, 19(3):355-369.

Qin, J., Liu, L., Shen, H., and Hu, D. (2020). Uniform pooling for graph networks. Applied Sciences, 10(18):6287.

Quiring, B. and Vassilevski, P. S. (2020). Multilevel graph embedding. Numerical Linear Algebra with Applications, SPECIAL ISSUE:e2326.

Ragesh, R., Sellamanickam, S., Iyer, A., Bairi, R., and Lingam, V. (2020). Hetegen: Heterogeneous graph convolutional networks for text classification. arXiv preprint arXiv:2008.12842, 2008.12842.

Ren, X., He, W., Qu, M., Voss, C. R., Ji, H., and Han, J. (2016). Label noise reduction in entity typing by heterogeneous partial-label embedding. In Proceedings of the 22nd ACM SIGKDD international conference on Knowledge discovery and data mining, pages 1825-1834. ACM.

Ribeiro, L. F., Saverese, P. H., and Figueiredo, D. R. (2017). struc2vec: Learning node representations from structural identity. In Proceedings of the 23rd ACM SIGKDD International Conference on Knowledge Discovery and Data Mining, volume 23, pages 385-394. ACM, MIT press.

Rissanen, J. (1978). Modeling by shortest data description. Automatica, 14(5):465-471.

Robins, G., Snijders, T., Wang, P., Handcock, M., and Pattison, P. (2007). Recent developments in exponential random graph (p*) models for social networks. Social networks, 29(2):192-215.

Rokka Chhetri, S. and Al Faruque, M. A. (2020). Dynamic Graph Embedding, pages 209-229. Springer International Publishing, Cham.

Rossi, E., Chamberlain, B., Frasca, F., Eynard, D., Monti, F., and Bronstein, M. (2020a). Temporal graph networks for deep learning on dynamic graphs. arXiv preprint arXiv:2006.10637, 2006.10637.

Rossi, E., Frasca, F., Chamberlain, B., Eynard, D., Bronstein, M., and Monti, F. (2020b). Sign: Scalable inception graph neural networks. arXiv preprint arXiv:2004.11198.

Roweis, S. T. and Saul, L. K. (2000). Nonlinear dimensionality reduction by locally linear embedding. science, 290(5500):2323-2326.

Rozemberczki, B., Davies, R., Sarkar, R., and Sutton, C. (2018). Gemsec: Graph embedding with self clustering. arXiv preprint arXiv:1802.03997, 1802.03997.

Rozemberczki, B. and Sarkar, R. (2018). Fast sequence-based embedding with diffusion graphs. In International Workshop on Complex Networks, pages 99-107. Springer International Publishing.

Sahu, S. K., Christopoulou, F., Miwa, M., and Ananiadou, S. (2019). Inter-sentence relation extraction with document-level graph convolutional neural network. In Proceedings of the 57th Annual Meeting of the Association for Computational Linguistics, pages 4309-4316, Florence, Italy. Association for 
Computational Linguistics.

Salha, G., Hennequin, R., and Vazirgiannis, M. (2020). Simple and effective graph autoencoders with one-hop linear models.

Salim, A., Shiju, S., and Sumitra, S. (2020). Design of multi-view graph embedding using multiple kernel learning. Engineering Applications of Artificial Intelligence, 90:103534.

Scarselli, F., Gori, M., Tsoi, A. C., Hagenbuchner, M., and Monfardini, G. (2009). The graph neural network model. IEEE Transactions on Neural Networks, 20(1):61-80.

Sen, P., Namata, G. M., Bilgic, M., Getoor, L., Gallagher, B., and Eliassi-Rad, T. (2008). Collective classification in network data. AI Magazine, 29(3):93-106.

Serra, A., Greco, D., and Tagliaferri, R. (2015). Impact of different metrics on multi-view clustering. In 2015 International Joint Conference on Neural Networks (IJCNN), pages 1-8. IEEE, IEEE.

Sevgili, Ö., Panchenko, A., and Biemann, C. (2019). Improving neural entity disambiguation with graph embeddings. In Proceedings of the 57th Annual Meeting of the Association for Computational Linguistics: Student Research Workshop, pages 315-322, Florence, Italy. Association for Computational Linguistics.

Shang, J., Ma, T., Xiao, C., and Sun, J. (2019). Pre-training of graph augmented transformers for medication recommendation. CoRR, abs/1906.00346.

Shavitt, Y. and Tankel, T. (2008). Hyperbolic embedding of internet graph for distance estimation and overlay construction. IEEE/ACM Transactions on Networking, 16(1):25-36.

Shaw, B. and Jebara, T. (2009). Structure preserving embedding. In Proceedings of the 26th Annual International Conference on Machine Learning, pages 937-944. ACM.

Shen, Z., Zhang, Y.-H., Han, K., Nandi, A. K., Honig, B., and Huang, D.-S. (2017). mirna-disease association prediction with collaborative matrix factorization. Complexity, 2017.

Shervashidze, N., Schweitzer, P., Leeuwen, E. J. v., Mehlhorn, K., and Borgwardt, K. M. (2011). Weisfeiler-lehman graph kernels. Journal of Machine Learning Research, 12(Sep):2539-2561.

Shi, J. and Malik, J. (2000). Normalized cuts and image segmentation. Departmental Papers (CIS), page 107.

Shi, L., Zhang, Y., Cheng, J., and Lu, H. (2019). Skeleton-based action recognition with directed graph neural networks. In The IEEE Conference on Computer Vision and Pattern Recognition (CVPR). IEEE.

Shi, M., Tang, Y., and Zhu, X. (2020a). Topology and content co-alignment graph convolutional learning.

Shi, R., Liang, T., Peng, H., Jiang, L., and Dai, Q. (2020b). Heam: Heterogeneous network embedding with automatic meta-path construction. In Li, G., Shen, H. T., Yuan, Y., Wang, X., Liu, H., and Zhao, X., editors, Knowledge Science, Engineering and Management, pages 304-315, Cham. Springer International Publishing.

Shuman, D. I., Narang, S. K., Frossard, P., Ortega, A., and Vandergheynst, P. (2013). The emerging field of signal processing on graphs: Extending high-dimensional data analysis to networks and other irregular domains. IEEE signal processing magazine, 30(3):83-98.

Si, C., Chen, W., Wang, W., Wang, L., and Tan, T. (2019). An attention enhanced graph convolutional 1 stm network for skeleton-based action recognition. In The IEEE Conference on Computer Vision and Pattern Recognition (CVPR). IEEE.

Sinha, A., Shen, Z., Song, Y., Ma, H., Eide, D., Hsu, B.-J. P., and Wang, K. (2015). An overview of microsoft academic service (mas) and applications. In Proceedings of the 24th International Conference on World Wide Web, WWW '15 Companion, page 243-246, New York, NY, USA. Association for Computing Machinery.

Socher, R., Chen, D., Manning, C. D., and Ng, A. (2013). Reasoning with neural tensor networks for knowledge base completion. In Advances in neural information processing systems, pages 926-934. MIT press.

Song, L. (2018). Structure2vec: Deep learning for security analytics over graphs.

Song, W., Xiao, Z., Wang, Y., Charlin, L., Zhang, M., and Tang, J. (2019). Session-based social recommendation via dynamic graph attention networks. CoRR, abs/1902.09362.

Srinivas, V. and Mitra, P. (2016). Applications of Link Prediction, chapter 5, pages 57-61. Springer International Publishing, Cham.

Stanovsky, G., Gruhl, D., and Mendes, P. (2017). Recognizing mentions of adverse drug reaction in social media using knowledge-infused recurrent models. In Proceedings of the 15th Conference of the European Chapter of the Association for Computational Linguistics: Volume 1, Long Papers, pages 
142-151, Valencia, Spain. Association for Computational Linguistics.

Su, C., Tong, J., Zhu, Y., Cui, P., and Wang, F. (2020). Network embedding in biomedical data science. Briefings in bioinformatics, 21(1):182-197.

Sun, F.-Y., Hoffmann, J., and Tang, J. (2019a). Infograph: Unsupervised and semi-supervised graphlevel representation learning via mutual information maximization. arXiv preprint arXiv:1908.01000, 1908.01000 .

Sun, J., Bandyopadhyay, B., Bashizade, A., Liang, J., Sadayappan, P., and Parthasarathy, S. (2019b). Atp: Directed graph embedding with asymmetric transitivity preservation. In Proceedings of the AAAI Conference on Artificial Intelligence, volume 33, pages 265-272. AAAI.

Sun, L., Wang, J., Yu, P. S., and Li, B. (2018a). Adversarial attack and defense on graph data: A survey. arXiv preprint arXiv:1812.10528, 1812.10528.

Sun, M., Tang, J., Li, H., Li, B., Xiao, C., Chen, Y., and Song, D. (2018b). Data poisoning attack against unsupervised node embedding methods. arXiv preprint arXiv:1810.12881, 1810.12881.

Sun, X., Guo, J., Ding, X., and Liu, T. (2016). A general framework for content-enhanced network representation learning. arXiv preprint arXiv:1610.02906, 1610.02906.

Tang, J. and Liu, H. (2012). Unsupervised feature selection for linked social media data. In Proceedings of the 18th ACM SIGKDD International Conference on Knowledge Discovery and Data Mining, KDD '12, pages 904-912, New York, NY, USA. ACM.

Tang, J., Liu, J., Zhang, M., and Mei, Q. (2016a). Visualizing large-scale and high-dimensional data. In Proceedings of the 25th international conference on world wide web, volume 25, pages 287-297. International World Wide Web Conferences Steering Committee, ACM.

Tang, J., Qu, M., Wang, M., Zhang, M., Yan, J., and Mei, Q. (2015). Line: Large-scale information network embedding. In Proceedings of the 24th IC on $W W W$, volume 24, pages 1067-1077. ACM, ACM.

Tang, L. and Liu, H. (2009). Relational learning via latent social dimensions. In Proceedings of the 15th ACM SIGKDD international conference on Knowledge discovery and data mining, volume 15, pages 817-826. ACM.

Tang, L. and Liu, H. (2011). Leveraging social media networks for classification. Data Mining and Knowledge Discovery, 23(3):447-478.

Tang, M., Nie, F., and Jain, R. (2016b). Capped lp-norm graph embedding for photo clustering. In Proceedings of the 2016 ACM on Multimedia Conference, pages 431-435. ACM.

Tenenbaum, J. B., De Silva, V., and Langford, J. C. (2000). A global geometric framework for nonlinear dimensionality reduction. science, 290(5500):2319-2323.

Teng, X. and Liu, J. (2020). Atrributed graph embedding based on multiobjective evolutionary algorithm for overlapping community detection. In 2020 IEEE Congress on Evolutionary Computation (CEC), pages 1-8. IEEE.

Tian, F., Gao, B., Cui, Q., Chen, E., and Liu, T.-Y. (2014). Learning deep representations for graph clustering. In $A A A I$, pages 1293-1299.

Tian, Y., Hankins, R. A., and Patel, J. M. (2008). Efficient aggregation for graph summarization. In Proceedings of the 2008 ACM SIGMOD international conference on Management of data, pages 567-580. ACM.

Toivonen, H., Zhou, F., Hartikainen, A., and Hinkka, A. (2011). Compression of weighted graphs. In Proceedings of the 17th ACM SIGKDD international conference on Knowledge discovery and data mining, pages 965-973. ACM.

Trouillon, T., Dance, C. R., Gaussier, É., Welbl, J., Riedel, S., and Bouchard, G. (2017). Knowledge graph completion via complex tensor factorization. The Journal of Machine Learning Research, 18(1):4735-4772.

Trouillon, T. and Nickel, M. (2017). Complex and holographic embeddings of knowledge graphs: a comparison. arXiv preprint arXiv:1707.01475, 1707.01475.

Tsitsulin, A., Mottin, D., Karras, P., and Müller, E. (2018). Verse: Versatile graph embeddings from similarity measures. In Proceedings of the 2018 World Wide Web Conference, pages 539-548.

Tsitsulin, A., Munkhoeva, M., and Perozzi, B. (2020). Just slaq when you approximate: Accurate spectral distances for web-scale graphs. In Proceedings of The Web Conference 2020, pages 2697-2703.

Tu, C., Liu, H., Liu, Z., and Sun, M. (2017). Cane: Context-aware network embedding for relation modeling. In Proceedings of the 55th Annual Meeting of the Association for Computational Linguistics 
(Volume 1: Long Papers), volume 1, pages 1722-1731.

Tu, C., Zhang, W., Liu, Z., Sun, M., et al. (2016). Max-margin deepwalk: Discriminative learning of network representation. In IJCAI, volume 2016, pages 3889-3895. International Joint Conferences on Artificial Intelligence Organization.

Vashishth, S., Bhandari, M., Yadav, P., Rai, P., Bhattacharyya, C., and Talukdar, P. (2019). Incorporating syntactic and semantic information in word embeddings using graph convolutional networks. In Proceedings of the 57th Annual Meeting of the Association for Computational Linguistics, pages 3308-3318, Florence, Italy. Association for Computational Linguistics.

Vaswani, A., Shazeer, N., Parmar, N., Uszkoreit, J., Jones, L., Gomez, A. N., Kaiser, Ł., and Polosukhin, I. (2017). Attention is all you need. In Advances in neural information processing systems, pages 5998-6008. MIT press.

Veličković, P., Cucurull, G., Casanova, A., Romero, A., Lio, P., and Bengio, Y. (2017). Graph attention networks. arXiv preprint arXiv:1710.10903, 1710.10903.

Veyseh, A. P. B., Nguyen, T. H., and Dou, D. (2019). Graph based neural networks for event factuality prediction using syntactic and semantic structures. CoRR, abs/1907.03227.

Vishwanathan, S. V. N., Schraudolph, N. N., Kondor, R., and Borgwardt, K. M. (2010). Graph kernels. Journal of Machine Learning Research, 11(Apr):1201-1242.

Wang, D., Cui, P., and Zhu, W. (2016). Structural deep network embedding. In Proceedings of the 22nd ACM SIGKDD international conference on Knowledge discovery and data mining, pages 1225-1234. ACM.

Wang, M. (2017). Predicting rich drug-drug interactions via biomedical knowledge graphs and text jointly embedding. arXiv, 1712.08875:arXiv-1712.

Wang, P., Wu, Q., Cao, J., Shen, C., Gao, L., and van den Hengel, A. (2018). Neighbourhood watch: Referring expression comprehension via language-guided graph attention networks. CoRR, abs/1812.04794.

Wang, P., Xu, B., Wu, Y., and Zhou, X. (2015). Link prediction in social networks: the state-of-the-art. Science China Information Sciences, 58(1):1-38.

Wang, Q., Mao, Z., Wang, B., and Guo, L. (2017a). Knowledge graph embedding: A survey of approaches and applications. IEEE Transactions on Knowledge and Data Engineering, 29(12):2724-2743.

Wang, S., Huang, E., Cairns, J., Peng, J., Wang, L., and Sinha, S. (2019a). Identification of pathways associated with chemosensitivity through network embedding. PLoS computational biology, 15(3):e1006864.

Wang, S., Qu, M., and Peng, J. (2017b). Prosnet: Integrating homology with molecular networks for protein function prediction. In PACIFIC SYMPOSIUM ON BIOCOMPUTING 2017, pages 27-38. World Scientific, World Scientific.

Wang, S., Tang, J., Aggarwal, C., Chang, Y., and Liu, H. (2017c). Signed network embedding in social media. In Proceedings of the 2017 SIAM international conference on data mining, pages 327-335. SIAM.

Wang, X., Bo, D., Shi, C., Fan, S., Ye, Y., and Yu, P. S. (2020). A survey on heterogeneous graph embedding: Methods, techniques, applications and sources. arXiv preprint arXiv:2011.14867.

Wang, X., Cui, P., Wang, J., Pei, J., Zhu, W., and Yang, S. (2017d). Community preserving network embedding. In $A A A I$, pages 203-209.

Wang, Z., Ye, X., Wang, C., Cui, J., and Yu, P. (2020). Network embedding with completely-imbalanced labels. IEEE Transactions on Knowledge and Data Engineering, pages 1-1.

Wang, Z., Zhang, J., Feng, J., and Chen, Z. (2014). Knowledge graph embedding by translating on hyperplanes. In Aaai, volume 14, pages 1112-1119. Citeseer, AAAI.

Wang, Z., Zheng, L., Li, Y., and Wang, S. (2019b). Linkage based face clustering via graph convolution network. In The IEEE Conference on Computer Vision and Pattern Recognition (CVPR). IEEE.

Waradpande, V., Kudenko, D., and Khosla, M. (2020). Deep reinforcement learning with graph-based state representations. arXiv preprint arXiv:2004.13965, 2004.13965.

Watts, D. J. and Strogatz, S. H. (1998). Collective dynamics of 'small-world'networks. nature, 393(6684):440-442.

Wei, X., Xu, L., Cao, B., and Yu, P. S. (2017). Cross view link prediction by learning noise-resilient representation consensus. In Proceedings of the 26th International Conference on World Wide Web, pages 1611-1619. International World Wide Web Conferences Steering Committee.

Weng, Z., Zhang, W., and Dou, W. (2020). Adversarial attention-based variational graph autoencoder. 
IEEE Access, 8:152637-152645.

White, S. and Smyth, P. (2005). A spectral clustering approach to finding communities in graphs. In Proceedings of the 2005 SIAM international conference on data mining, pages 274-285. SIAM.

Wilson, R. C., Hancock, E. R., Pekalska, E., and Duin, R. P. (2014). Spherical and hyperbolic embeddings of data. IEEE transactions on pattern analysis and machine intelligence, 36(11):2255-2269.

Wold, S., Esbensen, K., and Geladi, P. (1987). Principal component analysis. Chemometrics and intelligent laboratory systems, 2(1-3):37-52.

Wu, C., Zhou, Y., Tan, L., and Teng, C. (2020). Link prediction based on graph embedding method in unweighted networks. In 2020 39th Chinese Control Conference (CCC), pages 736-741.

Wu, Q., Zhang, H., Gao, X., He, P., Weng, P., Gao, H., and Chen, G. (2019a). Dual graph attention networks for deep latent representation of multifaceted social effects in recommender systems. CoRR, abs/1903.10433.

Wu, Z., Pan, S., Chen, F., Long, G., Zhang, C., and Yu, P. S. (2019b). A comprehensive survey on graph neural networks. arXiv preprint arXiv:1901.00596, 1901.00596.

Xiaojin, Z. and Zoubin, G. (2002). Learning from labeled and unlabeled data with label propagation. Tech. Rep., Technical Report CMU-CALD-02-107, Carnegie Mellon University.

Xie, M., Yin, H., Wang, H., Xu, F., Chen, W., and Wang, S. (2016). Learning graph-based poi embedding for location-based recommendation. In Proceedings of the 25th ACM International on Conference on Information and Knowledge Management, volume 25, pages 15-24. ACM, ACM.

Xiong, C., Power, R., and Callan, J. (2017). Explicit semantic ranking for academic search via knowledge graph embedding. In Proceedings of the 26th international conference on world wide web, pages 1271-1279. International World Wide Web Conferences Steering Committee.

Xu, L., Wei, X., Cao, J., and Yu, P. S. (2017). Embedding of embedding (eoe): Joint embedding for coupled heterogeneous networks. In Proceedings of the Tenth ACM International Conference on Web Search and Data Mining, pages 741-749. ACM.

Xu, X., Yuruk, N., Feng, Z., and Schweiger, T. A. (2007). Scan: a structural clustering algorithm for networks. In Proceedings of the 13th ACM SIGKDD international conference on Knowledge discovery and data mining, pages 824-833. ACM.

Xue, Z., Li, G., Wang, S., Zhang, C., Zhang, W., and Huang, Q. (2015). Gomes: A group-aware multiview fusion approach towards real-world image clustering. In 2015 IEEE International Conference on Multimedia and Expo (ICME), pages 1-6. IEEE, IEEE.

Yamanishi, Y., Araki, M., Gutteridge, A., Honda, W., and Kanehisa, M. (2008). Prediction of drugtarget interaction networks from the integration of chemical and genomic spaces. Bioinformatics, 24(13):i232-i240.

Yamanishi, Y., Kotera, M., Moriya, Y., Sawada, R., Kanehisa, M., and Goto, S. (2014). Dinies: drugtarget interaction network inference engine based on supervised analysis. Nucleic acids research, 42(W1):W39-W45.

Yan, B. and Wang, C. (2020). Graphae: Adaptive embedding across graphs. In 2020 IEEE 36th International Conference on Data Engineering (ICDE), pages 1958-1961. IEEE.

Yan, Z., Ge, J., Wu, Y., Li, L., and Li, T. (2020). Automatic virtual network embedding: A deep reinforcement learning approach with graph convolutional networks. IEEE Journal on Selected Areas in Communications, 38(6):1040-1057.

Yanardag, P. and Vishwanathan, S. (2015). Deep graph kernels. In Proceedings of the 21th ACM SIGKDD International Conference on Knowledge Discovery and Data Mining, pages 1365-1374. ACM.

Yang, B., Yih, W.-t., He, X., Gao, J., and Deng, L. (2014). Embedding entities and relations for learning and inference in knowledge bases. arXiv preprint arXiv:1412.6575, 1412.6575.

Yang, C., Liu, Z., Zhao, D., Sun, M., and Chang, E. (2015). Network representation learning with rich text information. In Twenty-Fourth International Joint Conference on Artificial Intelligence, pages 2111-2117.

Yang, L., Xiao, Z., Jiang, W., Wei, Y., Hu, Y., and Wang, H. (2020). Dynamic heterogeneous graph embedding using hierarchical attentions. In Jose, J. M., Yilmaz, E., Magalhães, J., Castells, P., Ferro, N., Silva, M. J., and Martins, F., editors, Advances in Information Retrieval, pages 425-432, Cham. Springer International Publishing.

Yang, L., Zhan, X., Chen, D., Yan, J., Loy, C. C., and Lin, D. (2019). Learning to cluster faces on an affinity graph. In The IEEE Conference on Computer Vision and Pattern Recognition (CVPR). IEEE. 
Yang, Y. and Wang, H. (2018). Multi-view clustering: A survey. Big Data Mining and Analytics, 1(2):83-107.

Yang, Z., Cohen, W. W., and Salakhutdinov, R. (2016a). Revisiting semi-supervised learning with graph embeddings. arXiv preprint arXiv:1603.08861, 1603.08861.

Yang, Z., Tang, J., and Cohen, W. W. (2016b). Multi-modal bayesian embeddings for learning social knowledge graphs. In IJCAI, pages 2287-2293.

Ye, Y., Hou, S., Chen, L., Lei, J., Wan, W., Wang, J., Xiong, Q., and Shao, F. (2019). Out-of-sample node representation learning for heterogeneous graph in real-time android malware detection. In Proceedings of the Twenty-Eighth International Joint Conference on Artificial Intelligence, IJCAI-19, pages 4150-4156. International Joint Conferences on Artificial Intelligence Organization.

Ying, R., Bourgeois, D., You, J., Zitnik, M., and Leskovec, J. (2019). Gnn explainer: A tool for post-hoc explanation of graph neural networks. arXiv preprint arXiv:1903.03894, 1903.03894.

Ying, R., He, R., Chen, K., Eksombatchai, P., Hamilton, W. L., and Leskovec, J. (2018a). Graph convolutional neural networks for web-scale recommender systems. arXiv preprint arXiv:1806.01973, 1806.01973 .

Ying, Z., You, J., Morris, C., Ren, X., Hamilton, W., and Leskovec, J. (2018b). Hierarchical graph representation learning with differentiable pooling. In Advances in Neural Information Processing Systems, pages 4805-4815. MIT press.

You, J., Ying, R., Ren, X., Hamilton, W. L., and Leskovec, J. (2018). Graphrnn: A deep generative model for graphs. arXiv preprint arXiv:1802.08773, 1802.08773.

Yu, W., Zheng, C., Cheng, W., Aggarwal, C. C., Song, D., Zong, B., Chen, H., and Wang, W. (2018). Learning deep network representations with adversarially regularized autoencoders. In Proceedings of the 24th ACM SIGKDD International Conference on Knowledge Discovery \& Data Mining, pages 2663-2671. ACM.

Yuan, H. and Ji, S. (2020). Structpool: Structured graph pooling via conditional random fields. In International Conference on Learning Representations.

Yuan, S., Wu, X., and Xiang, Y. (2017). Sne: signed network embedding. In Pacific-Asia conference on knowledge discovery and data mining, pages 183-195. Springer.

Zeng, H., Zhou, H., Srivastava, A., Kannan, R., and Prasanna, V. K. (2019). Graphsaint: Graph sampling based inductive learning method. CoRR, abs/1907.04931.

Zhai, S. and Zhang, Z. (2015). Dropout training of matrix factorization and autoencoder for link prediction in sparse graphs. In Proceedings of the 2015 SIAM International Conference on Data Mining, pages 451-459. SIAM, SIAM.

Zhang, C., Ren, M., and Urtasun, R. (2019a). Graph hypernetworks for neural architecture search. In International Conference on Learning Representations. ICLR.

Zhang, D., Dai, X., Wang, X., Wang, Y., and Davis, L. S. (2018a). MAN: moment alignment network for natural language moment retrieval via iterative graph adjustment. CoRR, abs/1812.00087.

Zhang, D., Yin, J., Zhu, X., and Zhang, C. (2016a). Collective classification via discriminative matrix factorization on sparsely labeled networks. In Proceedings of the 25th ACM International on Conference on Information and Knowledge Management, pages 1563-1572. ACM.

Zhang, D., Yin, J., Zhu, X., and Zhang, C. (2016b). Homophily, structure, and content augmented network representation learning. In 2016 IEEE 16th International Conference on Data Mining (ICDM), pages 609-618. IEEE.

Zhang, H., Shang, X., Luan, H., Wang, M., and Chua, T.-S. (2017). Learning from collective intelligence: Feature learning using social images and tags. ACM transactions on multimedia computing, communications, and applications (TOMM), 13(1):1.

Zhang, H., Shang, X., Luan, H., Yang, Y., and Chua, T.-S. (2015). Learning features from large-scale, noisy and social image-tag collection. In Proceedings of the 23rd ACM international conference on Multimedia, pages 1079-1082. ACM.

Zhang, H., Zheng, T., Gao, J., Miao, C., Su, L., Li, Y., and Ren, K. (2019b). Towards data poisoning attack against knowledge graph embedding. arXiv preprint arXiv:1904.12052, 1904.12052.

Zhang, H., Zhou, J., and Li, R. (2020). Enhanced unsupervised graph embedding via hierarchical graph convolution network. Mathematical Problems in Engineering, 2020.

Zhang, J., Shi, X., Zhao, S., and King, I. (2019c). STAR-GCN: stacked and reconstructed graph convolutional networks for recommender systems. CoRR, abs/1905.13129. 
Zhang, Q. and Wang, H. (2016). Not all links are created equal: An adaptive embedding approach for social personalized ranking. In Proceedings of the 39th International ACM SIGIR conference on Research and Development in Information Retrieval, pages 917-920. ACM.

Zhang, W., Fang, Y., Liu, Z., Wu, M., and Zhang, X. (2020). mg2vec: Learning relationship-preserving heterogeneous graph representations via metagraph embedding. IEEE Transactions on Knowledge and Data Engineering, pages 1-1.

Zhang, W., Shang, R., and Jiao, L. (2020a). Complex network graph embedding method based on shortest path and moea/d for community detection. Applied Soft Computing, page 106764.

Zhang, Y. and Chen, X. (2018). Explainable recommendation: A survey and new perspectives. arXiv preprint arXiv:1804.11192, 1804.11192.

Zhang, Y., Wang, D., and Zhang, Y. (2019d). Neural IR meets graph embedding: A ranking model for product search. CoRR, abs/1901.08286.

Zhang, Z., Bu, J., Ester, M., Zhang, J., Yao, C., Li, Z., and Wang, C. (2020b). Learning temporal interaction graph embedding via coupled memory networks. In Proceedings of The Web Conference 2020, WWW '20, page 3049-3055, New York, NY, USA. Association for Computing Machinery.

Zhang, Z., Cui, P., and Zhu, W. (2018b). Deep learning on graphs: A survey. CoRR, abs/1812.04202.

Zhao, G., Li, J., Wang, L., Qian, X., and Fu, Y. (2019). Graphseq2seq: Graph-sequence-to-sequence for neural machine translation.

Zhao, X., Chang, A., Sarma, A. D., Zheng, H., and Zhao, B. Y. (2013). On the embeddability of random walk distances. Proceedings of the VLDB Endowment, 6(14):1690-1701.

Zhao, Y., Liu, Z., and Sun, M. (2015). Representation learning for measuring entity relatedness with rich information. In Twenty-Fourth International Joint Conference on Artificial Intelligence, pages $1412-1418$.

Zhao, Z., Yang, Q., Cai, D., He, X., and Zhuang, Y. (2016). Expert finding for community-based question answering via ranking metric network learning. In IJCAI, pages 3000-3006.

Zheng, D., Ma, C., Wang, M., Zhou, J., Su, Q., Song, X., Gan, Q., Zhang, Z., and Karypis, G. (2020a). Distdgl: Distributed graph neural network training for billion-scale graphs. arXiv preprint arXiv:2010.05337, 2010.05337.

Zheng, V. W., Cavallari, S., Cai, H., Chang, K. C.-C., and Cambria, E. (2016). From node embedding to community embedding. arXiv preprint arXiv:1610.09950, 1610.09950.

Zheng, W., Wang, D., and Song, F. (2020b). Opengraphgym: A parallel reinforcement learning framework for graph optimization problems. In Krzhizhanovskaya, V. V., Závodszky, G., Lees, M. H., Dongarra, J. J., Sloot, P. M. A., Brissos, S., and Teixeira, J., editors, Computational Science - ICCS 2020, pages 439-452, Cham. Springer International Publishing.

Zheng, X., Ding, H., Mamitsuka, H., and Zhu, S. (2013). Collaborative matrix factorization with multiple similarities for predicting drug-target interactions. In Proceedings of the 19th ACM SIGKDD international conference on Knowledge discovery and data mining, pages 1025-1033. ACM.

Zhong, J., Li, N., Kong, W., Liu, S., Li, T. H., and Li, G. (2019). Graph convolutional label noise cleaner: Train a plug-and-play action classifier for anomaly detection. CoRR, abs/1903.07256.

Zhong, J., Qiu, H., and Shi, B. (2020). Dynamics-preserving graph embedding for community mining and network immunization. Information, 11(5):250.

Zhou, C., Liu, Y., Liu, X., Liu, Z., and Gao, J. (2017). Scalable graph embedding for asymmetric proximity. In $A A A I$, pages 2942-2948.

Zhou, K., Michalak, T. P., Rahwan, T., Waniek, M., and Vorobeychik, Y. (2018). Adversarial link prediction in social networks. arXiv preprint arXiv:1809.08368, 1809.08368.

Zhou, S., Dai, X., Chen, H., Zhang, W., Ren, K., Tang, R., He, X., and Yu, Y. (2020). Interactive recommender system via knowledge graph-enhanced reinforcement learning. In Proceedings of the 43rd International ACM SIGIR Conference on Research and Development in Information Retrieval, SIGIR '20, page 179-188, New York, NY, USA. Association for Computing Machinery.

Zhou, Y., Cheng, H., and Yu, J. X. (2009). Graph clustering based on structural/attribute similarities. Proceedings of the VLDB Endowment, 2(1):718-729.

Zhu, H., Lin, Y., Liu, Z., Fu, J., Chua, T.-S., and Sun, M. (2019). Graph neural networks with generated parameters for relation extraction. In Proceedings of the 57th Annual Meeting of the Association for Computational Linguistics, pages 1331-1339, Florence, Italy. Association for Computational Linguistics. 
Zhu, S., Li, J., Peng, H., Wang, S., Yu, P. S., and He, L. (2020a). Adversarial directed graph embedding. arXiv preprint arXiv:2008.03667, 2008.03667.

Zhu, S., Yu, K., Chi, Y., and Gong, Y. (2007). Combining content and link for classification using matrix factorization. In Proceedings of the 30th annual international ACM SIGIR conference on Research and development in information retrieval, pages 487-494. ACM.

Zhu, S., Zhou, L., Pan, S., Zhou, C., Yan, G., and Wang, B. (2020b). Gssnn: Graph smoothing splines neural networks. In AAAI, pages 7007-7014. AAAI.

Zhu, Y., Xu, Y., Yu, F., Liu, Q., Wu, S., and Wang, L. (2020c). Deep graph contrastive representation learning.

Zitnik, M., Agrawal, M., and Leskovec, J. (2018). Modeling polypharmacy side effects with graph convolutional networks. Bioinformatics, 34(13):i457-i466.

Zitnik, M. and Leskovec, J. (2017). Predicting multicellular function through multi-layer tissue networks. Bioinformatics, 33(14):i190-i198.

Zitnik, M. and Zupan, B. (2016). Collective pairwise classification for multi-way analysis of disease and drug data. In Biocomputing 2016: Proceedings of the Pacific Symposium, pages 81-92. World Scientific, World Scientific.

Zong, N., Kim, H., Ngo, V., and Harismendy, O. (2017). Deep mining heterogeneous networks of biomedical linked data to predict novel drug-target associations. Bioinformatics, 33(15):2337-2344.

Zügner, D., Akbarnejad, A., and Günnemann, S. (2018). Adversarial attacks on classification models for graphs. arXiv preprint arXiv:1805.07984, 1805.07984.

Çelikkanat, A. and Malliaros, F. D. (2019). Exponential family graph embeddings. 